Recepción: 20 / 04 / 2017

Aceptación: 20 / 05 / 2017

Publicación: 15 / 06 / 2017

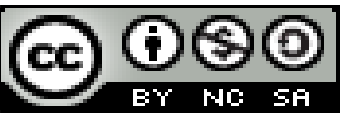

Ciencias Administrativas

Artículo de Investigación

\title{
Buenas prácticas de marketing del sector privado y su incidencia en la gestión pública en la coordinación zonal cinco del MIES
}
Good practices of marketing of the private sector and its incidence in the public management in the zonal coordination five of the MIES

\section{Práticas de marketing boas do setor privado e seu impacto sobre a governança na coordenação zonal Cinco MIES}

\author{
Teófilo R. Fernández-Bayas ${ }^{\mathrm{I}}$ \\ teofilo.fernandez@hotmail.com \\ Darío J. Arellano-Valencia II \\ dario.arellano@hotmail.com \\ Gabriela E. Medina-Pinoargote ${ }^{\text {III }}$ \\ gabriela.pinargote@hotmail.com
}

Correspondencia: teofilo.fernandez@hotmail.com

I. Ingeniero en Banca y Finanzas; Magister en Docencia y Gerencia en Educación Superior, Universidad de Guayaquil, Guayaquil, Ecuador.

II. Ingeniero en Banca y Finanzas; Magister en Administración de Empresas, Universidad Técnica de Babahoyo, Los Ríos, Ecuador.

III. Ingeniera Comercial; Diploma Superior en Pedagogías Innovadoras; Magister en Administración de Empresas, Universidad Técnica de Babahoyo, Los Ríos, Ecuador. 


\section{Resumen}

El presente trabajo aborda la gestión de los servicios públicos deportivos (náuticos) desde la perspectiva del marketing, y más concretamente el marketing social aplicado al deporte.

Realizamos un breve análisis del servicio público deportivo aportando algunas estrategias para mejorarlo. Entendemos que la gestión en los servicios públicos de deportes se debe basar en la calidad del servicio y para ellos presentarnos, después de abordar nuestra concepción de la calidad, las dimensiones que el usuario de las Escuelas del Mar de la Generadita Valenciana ha entendido que definen la calidad del servicio de estas escuelas. Por ultimo aportamos unas variables que creemos pueden mejorar la gestión de los servicios de las instituciones públicas.

Palabras clave: Gestión de servicios, Marketing y Deporte. 
Buenas prácticas de marketing del sector privado y su incidencia en la gestión pública MIES

\section{Abstract}

The present work deals with the management of public sports services (nautical) from the perspective of marketing, and more specifically social marketing applied to sport.

We make a brief analysis of the public sports service providing some strategies to improve it. We understand that management in public sports services must be based on the quality of the service and for them to present, after approaching our conception of quality, the dimensions that the users of the Sea Schools of the Valencian Generadita have understood that they define The quality of service of these schools. Finally, we provide some variables that we believe can improve the management of the services of public institutions.

Keywords: Service management, Marketing and Sports. 
Teófilo R. Fernández-Bayas; Darío J. Arellano-Valencia; Gabriela E. Medina-Pinoargote

\section{Resumo}

Este artigo trata da gestão de utilitários esportivos (água) a partir de uma perspectiva de marketing, e mais especificamente marketing social aplicada ao esporte.

Foi realizada uma breve análise do utilitário esportivo fornecendo algumas estratégias para melhoria. Entendemos que a gestão de serviços públicos de esportes deve ser baseada na qualidade do serviço e para eles a introduzir, depois de abordar a nossa concepção de qualidade, tamanho o usuário de Escolas Mar de Generadita Valenciana entendido que definem qualidade do serviço dessas escolas. Finalmente nós fornecemos algumas variáveis que podemos melhorar a gestão das instituições públicas serviço.

Palavras-chave: Gestão de Serviços, Marketing e Desporto. 


\section{Introducción.}

El presente proyecto de investigación pretende examinar la compleja problemática del proceso de las Buenas Prácticas del Marketing en la coordinación zonal cinco del Mies, para lo cual se presentará en detalle cada una de las particularidades que caracteriza al ciclo y que concita la preocupación de directivos, la búsqueda de una forma eficiente de lograr los objetivos planteados tanto en los usuarios internos como externos.

Por lo tanto, la preocupación reside en la manera en que la administración pública siempre se ha caracterizado por ejercer su gestión de manera ineficiente y centralizada, sin tomar en cuenta la opinión de los ciudadanos que claman, con todo derecho, una mejor atención en la cual puedan también ser partícipes de las decisiones que se tomen en beneficio de la colectividad.

Además, existe aún poca voluntad de cambio en algunos funcionarios públicos, existe pleno consenso en realizar constantes ajustes tanto en la estructura del Estado como en distintos procesos de la administración pública, iniciándose pequeños cambios como los expuestos en este trabajo, o a través de una gran reforma como la que se está gestionando actualmente en el país, con una administración que esté únicamente orientada a conocer y servir a su "cliente-ciudadano".

De tal manera, que la administración pública debe constituir una plataforma institucional que responda a los intereses generales de la sociedad, considerando al ciudadano como su principal "cliente".

Dichas políticas de marketing deben responder en su esencia a procesos sociales y de gestión a través de los cuales los individuos obtengan lo que necesitan por parte de quienes les proporcionarán satisfactores de valor, llámese Estado o entidades públicas. 
Además, en la investigación es establecer un proceso alterno al descrito, que sea ágil y tecnológicamente fundamentado cuyo impacto asevere las buenas prácticas de marketing, que nos permita incorporación en la gestión pública.

La investigación se inscribe en el marco de la Ingeniería Comercial, en este caso, mediante la gestión de Marketing, se propone estudiar la viabilidad de emplear la metodología de las prácticas de marketing usadas comúnmente por el sector privado para concebir un nuevo diseño, con mayores capacidades, flexible y eficiente, basado en los principios del marketing.

Es importante mencionar que los principios considerados como básicos en los modelos de gestión de marketing, tales como el marketing público, entre estos principios ocupa un lugar destacado la gestión con un enfoque basado en el servicio, que permite basarse en la elección de un público objetivo referencial para diseñar las políticas y así evaluar el rendimiento de las distintas actividades que se llevan a cabo, no solo consideradas de forma aislada, sino formando parte de un conjunto estrechamente interrelacionado, teorías que presentaremos en nuestro marco referencial.

El planteamiento del estudio, establecerá el marco de la problemática de la coordinación zonal cinco del mies, concretamente en el proceso de emisión de políticas de marketing, planteando interrogantes, relaciones entre variables, fines e interés en el tema.

El estudio de la solución, tiene como marco la normatividad de otros países, como Chile en que procesos de similar envergadura han sido transformados para operar convenientemente, con una legislación que se renueva en base a los avances de las nuevas tecnologías de la información y las comunicaciones. 


\section{Materiales y métodos}

Delimitación de la investigación.

\section{Temporal}

De enero del 2015 a junio del 2015

\section{Espacial}

Coordinación Zonal Cinco del Mies

Ubicación:

- Parroquia Camilo Ponce Enríquez.

- Ciudad de Babahoyo.

- Cantón Babahoyo.

- Provincia de Los Ríos

\section{Unidades de observación}

- Directivos:

- Administrativos:

- Usuarios:
(5) Cinco.

(27) Veinte y siete.

(500) Quinientos.

Los actores externos o usuarios lo constituyen las asociaciones, comunidades agrícolas y público en general que concurren a la prestación de los servicios que se brindan en la Coordinación Zonal Cinco del Mies.

La investigación propuesta busca, mediante la aplicación de la teoría y los conceptos básicos de las estrategias de las buenas prácticas de marketing aplicadas por las empresas privadas, para encontrar explicaciones a situaciones internas como es las carencias de procesos comunicacionales, se justifica por los problemas detectados en la Coordinación Zonal Cinco del Ministerio de inclusión económica y social que desmejora la atención del servicio y por ende su imagen institucional. 
Para lograr el cumplimiento de los objetivos de estudio, se acude al empleo de técnicas de investigación para medir la gestión de marketing en la Coordinación zonal cinco del Ministerio de Inclusión Económica y Social a través de la aplicación de entrevista y cuestionarios y su procesamiento, se busca conocer el grado de identificación de los procesos comunicacionales con los objetivos de la Institución que se tomen en beneficio de los usuarios tanto internos como externos.

De acuerdo con los objetivos de la investigación, su resultado permite encontrar alternativas concretas a problemas sociales y de gestión pública que influyen en los resultados de la efectividad y eficiencia de la institución.

La pertinencia de la presente investigación está relacionada a las Buenas Prácticas de Marketing que se ha implementado en el sector privado y que el mismo puede ser aplicado en la presente institución objeto de análisis, por lo tanto, es la adecuación de dicho modelo a la Coordinación Zonal Cinco del Mies.

\section{Resultados}

Pruebas estadísticas aplicadas en la verificación de las hipótesis.

Se determina un $100 \%$ que en la coordinación zonal cinco del Ministerio de Inclusión Económica y Social, las buenas prácticas de marketing no se aplica en su gestión pública, por lo que su proceso comunicacional se encuentra distanciado entre autoridades, empleados y trabajadores dando como resultado que los procesos internos se encuentren no vinculados con los usuarios, por ende, es necesario enfocarse en las buenas prácticas de marketing para mejorar su imagen institucional y su vinculamiento con los usuarios, lo que permitirá cubrir las actividades de los 
servicios que dispensa la institución y a su vez aumentar su cobertura de acción a favor de la comunidad Riosenses y poder cumplir los objetivos.

Se pudo comprobar que las Buenas Prácticas de Marketing en la Coordinación zonal cinco del Ministerio de Inclusión Económica y Social existe un desconocimiento de su utilidad e importancia, en cuanto, a su implementación para mejorar su atención al servicio y por ende sus procesos comunicacionales.

Análisis e interpretación de datos

Encuesta dirigida a los Directivos y empleados administrativos de la coordinación zonal cinco del MIES

\section{1.- ¿Cree usted conocer lo que es las Buenas Prácticas de Marketing?}

\begin{tabular}{|l|l|l|}
\hline \multicolumn{1}{|c|}{ Alternativas } & \multicolumn{1}{c|}{$\mathbf{N}^{\circ}$ encuestados } & \multicolumn{2}{c|}{ Porcentaje } \\
\hline Mucho & 5 & 18 \\
\hline Poco & 5 & 18 \\
\hline Nada & 17 & 64 \\
\hline TOTAL & 27 & 100 \\
\hline
\end{tabular}

\section{Cuadro 1}

Fuente: Parroquia Camilo Ponce Enríquez 


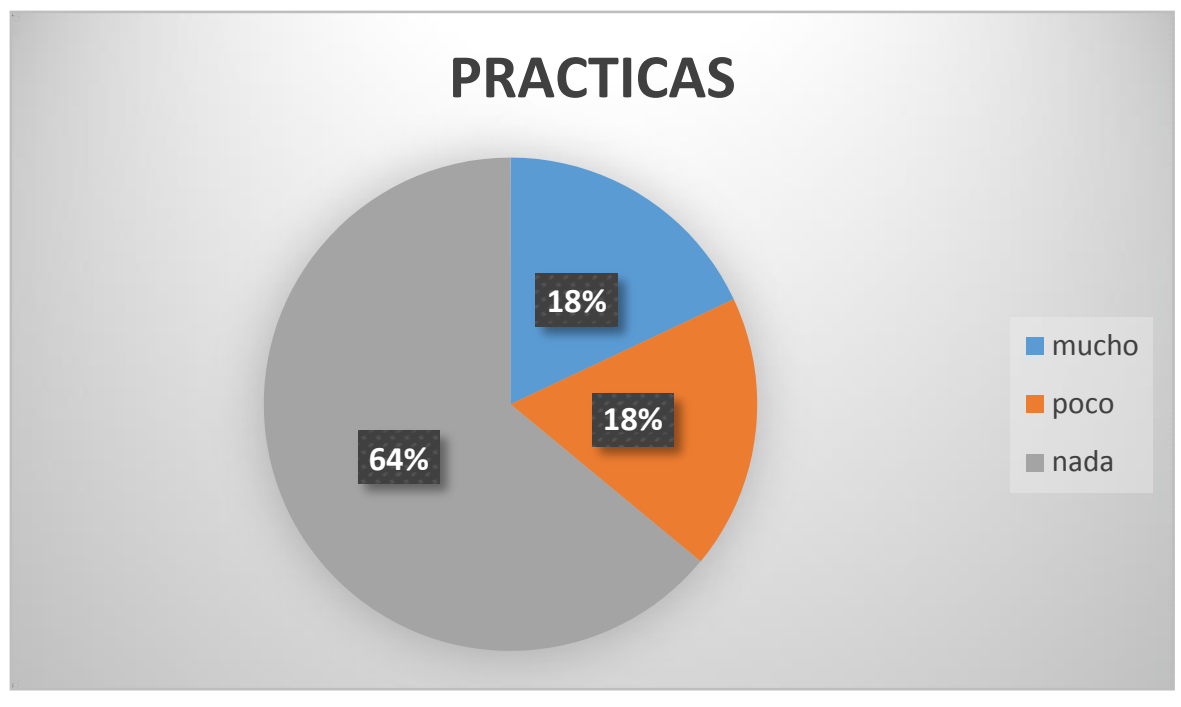

\section{Gráfico 1 Prácticas}

Fuente: Parroquia Camilo Ponce Enríquez

Análisis de datos En el instrumento aplicado a los directivos y empleados de la coordinación zona cinco del Mies, relacionada con las Buenas prácticas de marketing, los resultados obtenidos demuestran que un $64 \%$ desconocen lo que son las buenas prácticas de marketing, por lo que se puede interpretar que es necesario implementar esta temática

\section{2.- ¿Participó usted en la planificación de la gestión de marketing?}

\begin{tabular}{|l|l|l|}
\hline \multicolumn{1}{|c|}{ alternativas } & \multicolumn{1}{|c|}{$\mathbf{N}^{\circ}$ encuestados } & \multicolumn{1}{c|}{ porcentaje } \\
\hline Mucho & 2 & 08 \\
\hline Poco & 5 & 18 \\
\hline Nada & 20 & 74 \\
\hline TOTAL & 27 & 100 \\
\hline
\end{tabular}

\section{Cuadro 2}

Fuente: Parroquia Camilo Ponce Enríquez 


\section{PLANIFICACION}

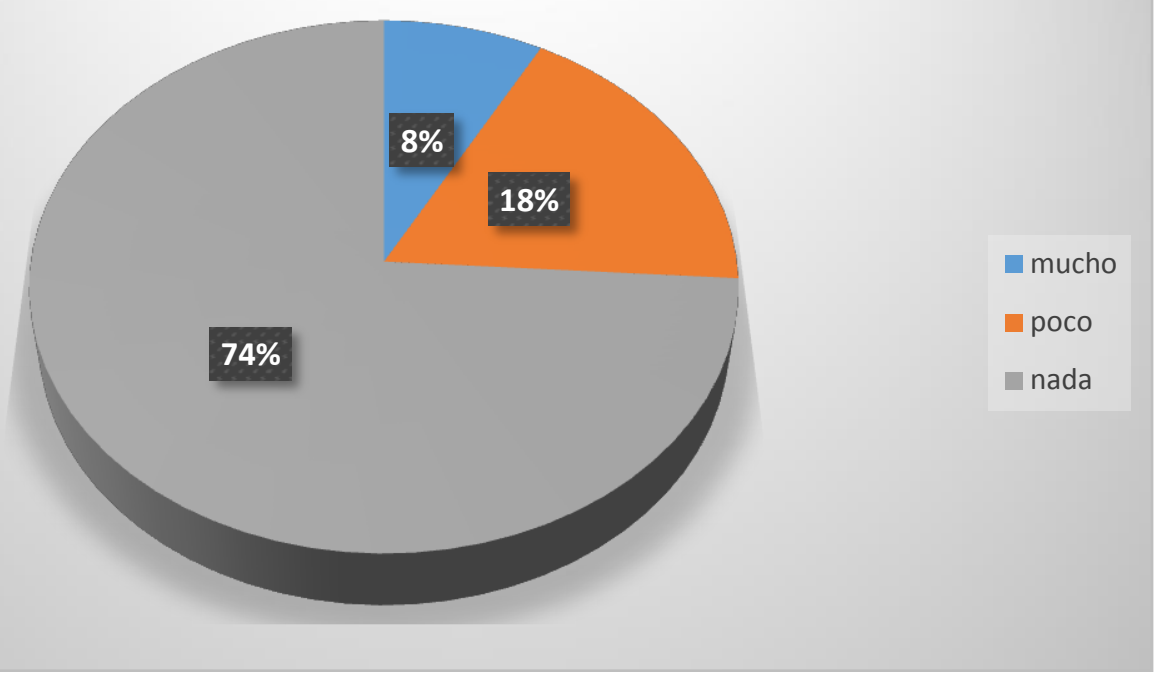

\section{Gráfico 2 Planificación}

Fuente: Parroquia Camilo Ponce Enríquez

Análisis de datos En la encuesta aplicada a los directivos y empleados de la coordinación zona cinco del Mies, relacionada con la participación en la planificación de la gestión de marketing, los resultados obtenidos demuestran que un 74\% no han sido considerados en la planificación, por lo que se puede deducir que es necesario involucrar a los empleados en la gestión de marketing.

\section{3.- ¿Considera usted que la gestión de marketing que mantiene la Coordinación Zonal cinco} del Mies es obsoleto?

\begin{tabular}{|l|l|l|}
\hline \multicolumn{1}{|c|}{ alternativas } & $\mathbf{N}^{\circ}$ encuestados & \multicolumn{2}{c|}{ Porcentaje } \\
\hline Mucho & 22 & 81 \\
\hline Poco & 3 & 11 \\
\hline Nada & 2 & 08 \\
\hline TOTAL & 27 & 100 \\
\hline
\end{tabular}

\section{Cuadro 3}

Fuente: Parroquia Camilo Ponce Enríquez 


\section{GESTION}

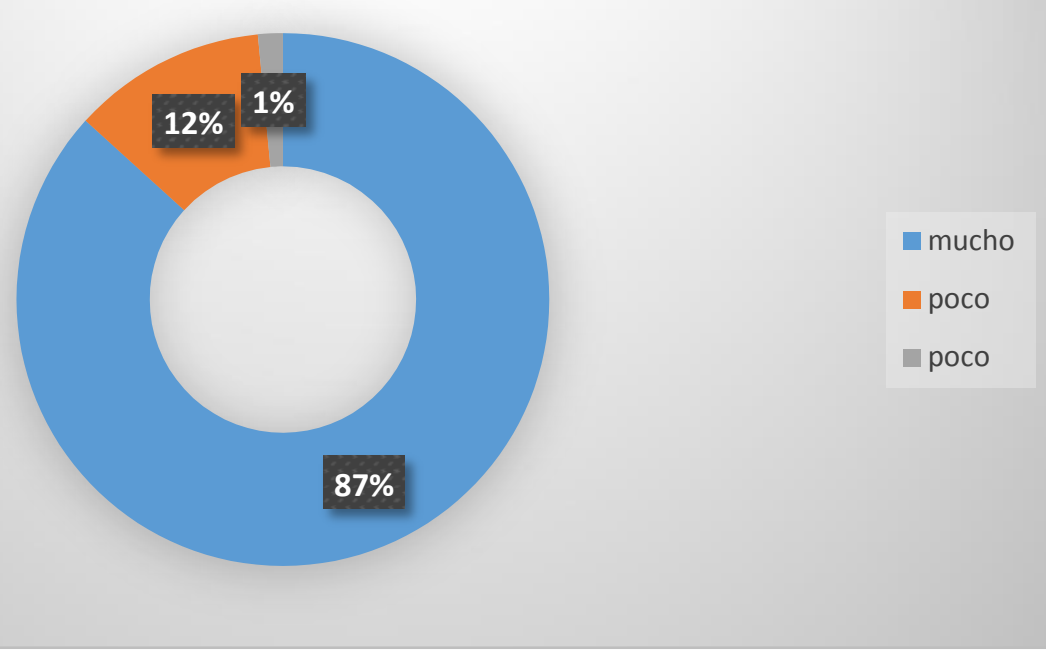

\section{Gráfico 3 Gestión}

Fuente: Parroquia Camilo Ponce Enríquez

Análisis de datos En la encuesta aplicada a los directivos y empleados de la coordinación zona cinco del Mies, relacionada con la gestión de marketing, los resultados obtenidos demuestran que un $81 \%$ consideran que la gestión de marketing está en un estado de mucha obsolescencia, por lo que se puede interpretar que amerita diseñar nuevas políticas de marketing hacer aplicadas en la institución.

\section{4.- ¿Usted ha participado en los diseños de las estrategias para mejorar la imagen}

\section{Institucional?}

\begin{tabular}{|l|l|l|}
\hline \multicolumn{1}{|c|}{ alternativas } & \multicolumn{1}{|c|}{$\mathbf{N}^{\circ}$ encuestados } & \multicolumn{1}{c|}{ porcentaje } \\
\hline Mucho & 3 & 11 \\
\hline Poco & 3 & 11 \\
\hline Nada & 21 & 78 \\
\hline TOTAL & 27 & 100 \\
\hline
\end{tabular}

\section{Cuadro 4}

Fuente: Parroquia Camilo Ponce Enríquez 


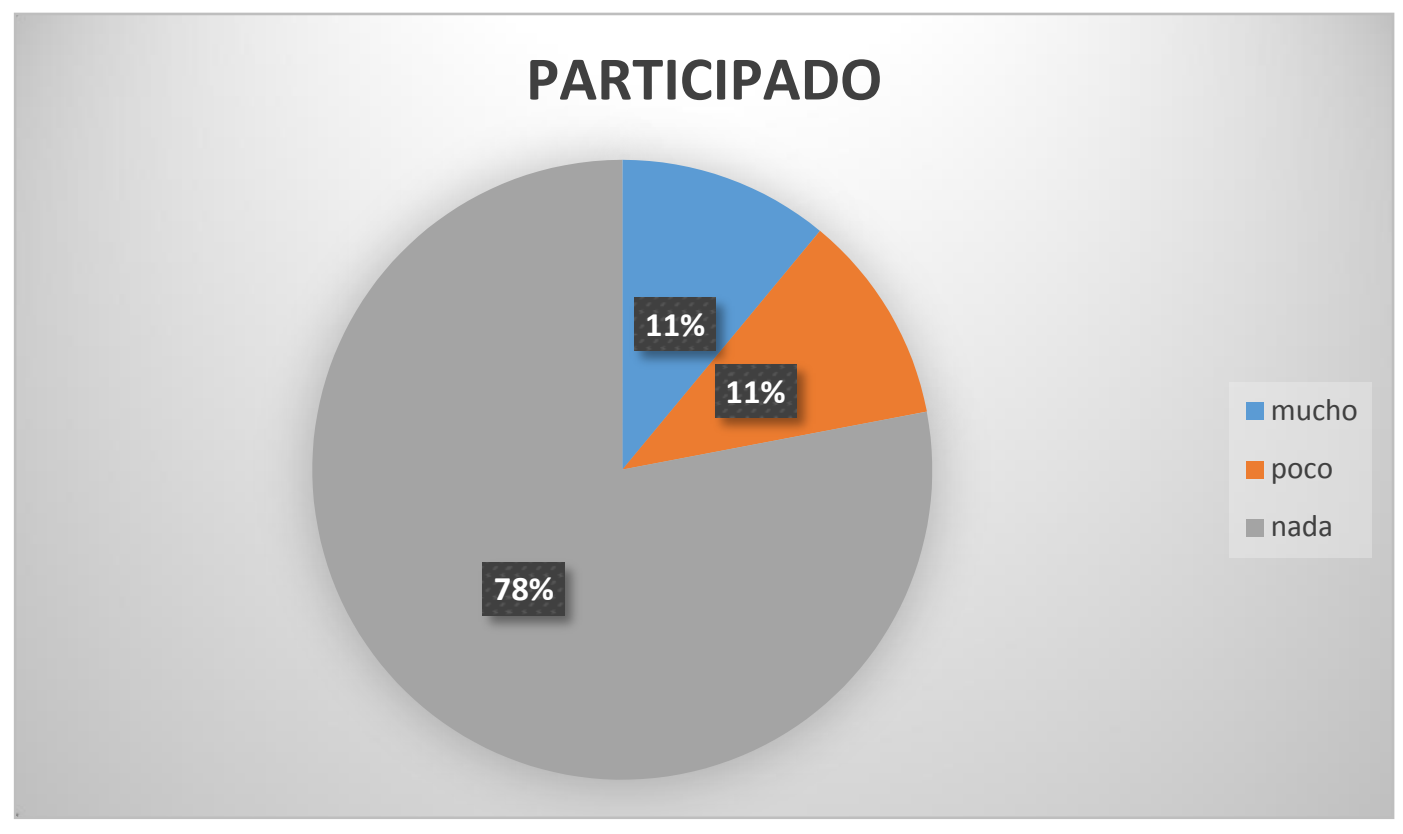

\section{Gráfico 4 Participado}

Fuente: Parroquia Camilo Ponce Enríquez

Análisis de datos En la encuesta aplicada a los directivos y empleados de la coordinación zonal cinco del Mies, relacionada con la participación en el diseño de estrategias para mejorar la imagen institucional, los resultados obtenidos demuestran que un $78 \%$ no han sido considerados en la participación de las estrategias, por lo que se puede deducir que es necesario involucrar a los empleados en la participación del diseño de estrategias. 


\section{5.- ¿Considera usted que la Coordinación Zonal cinco del Mies goza de buena imagen frente a} otras instituciones públicas?

\begin{tabular}{|l|l|l|}
\hline \multicolumn{1}{|c|}{ Alternativas } & \multicolumn{1}{c|}{$\mathbf{N}^{\circ}$ encuestados } & \multicolumn{1}{c|}{ Porcentaje } \\
\hline Mucho & 6 & 23 \\
\hline Poco & 5 & 18 \\
\hline Nada & 16 & 59 \\
\hline TOTAL & 27 & 100 \\
\hline
\end{tabular}

\section{Cuadro 5}

Fuente: Parroquia Camilo Ponce Enríquez

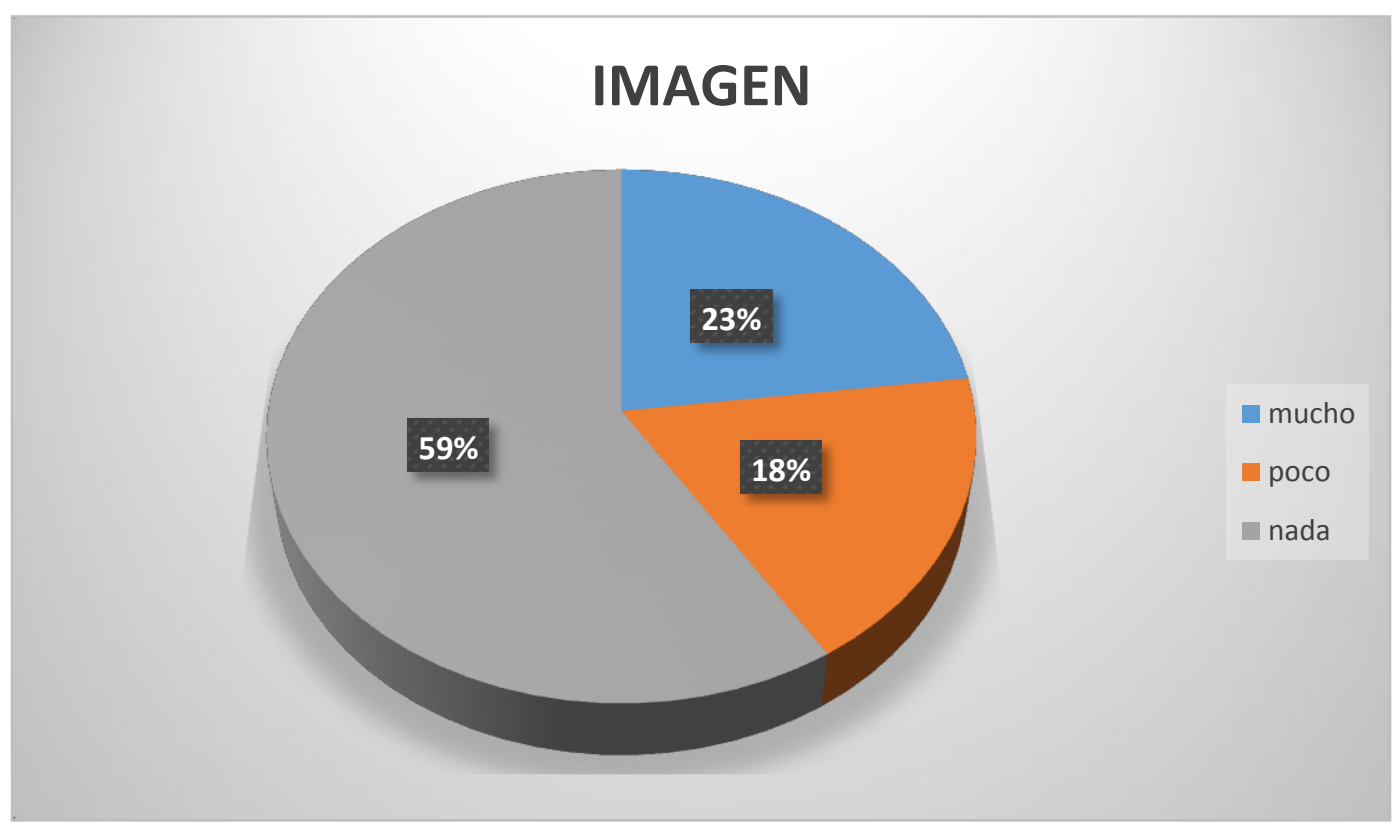

\section{Gráfico 5 Imagen}

Fuente: Parroquia Camilo Ponce Enríquez

Análisis de datos En la encuesta aplicada a los directivos y empleados de la coordinación zona cinco del Mies, relacionada con la buena imagen institucional comparado con otras instituciones públicas, los resultados obtenidos demuestran que un 59\% de los encuestados 
consideran que no tienen buena imagen frente a otras instituciones públicas, por lo que se puede interpretar que es necesario mejorar esa imagen institucional con procesos comunicacionales.

6.- ¿Creé que es necesario desarrollar nuevas estrategias de marketing que mejoren la imagen de la organización?

\begin{tabular}{|l|l|l|}
\hline \multicolumn{1}{|c|}{ Alternativas } & \multicolumn{1}{c|}{$\mathbf{N}^{\circ}$ encuestados } & Porcentaje \\
\hline Mucho & 20 & 74 \\
\hline Poco & 3 & 11 \\
\hline Nada & 4 & 13 \\
\hline TOTAL & 27 & 100 \\
\hline
\end{tabular}

\section{Cuadro 6}

Fuente: Parroquia Camilo Ponce Enríquez

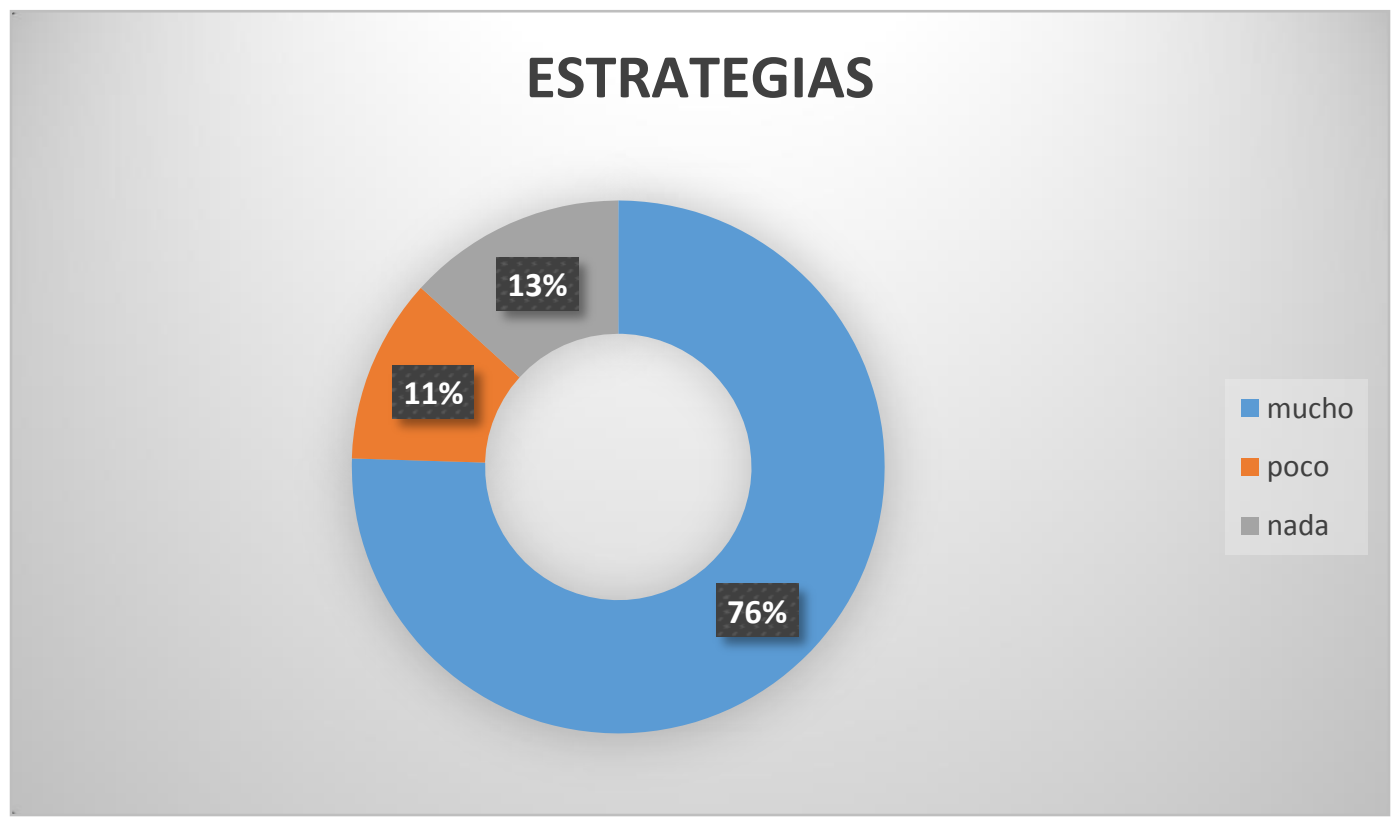

\section{Gráfico 6 Estrategias}

Fuente: Parroquia Camilo Ponce Enríquez

Análisis de datos En la encuesta aplicada a los directivos y empleados de la coordinación zonal cinco del Mies, relacionada con desarrollar nuevas estrategias de marketing para mejorar la imagen institucional, los resultados obtenidos demuestran que un $76 \%$ de los encuestados 
consideran que se deben desarrollar nuevas estrategias de marketing, por lo que se puede interpretar que es necesario estrategias actualizadas en la institución.

\section{7.- ¿Estima usted necesario que la Coordinación Zonal cinco del Mies cuente con una nueva gestión de marketing?}

\begin{tabular}{|l|l|l|}
\hline \multicolumn{1}{|c|}{ Alternativas } & \multicolumn{1}{c|}{$\mathbf{N}^{\circ}$ encuestados } & \multicolumn{1}{c|}{ porcentaje } \\
\hline Mucho & 21 & 74 \\
\hline Poco & 4 & 13 \\
\hline Nada & 2 & 13 \\
\hline TOTAL & 27 & 100 \\
\hline
\end{tabular}

Cuadro 7

Fuente: Parroquia Camilo Ponce Enríquez

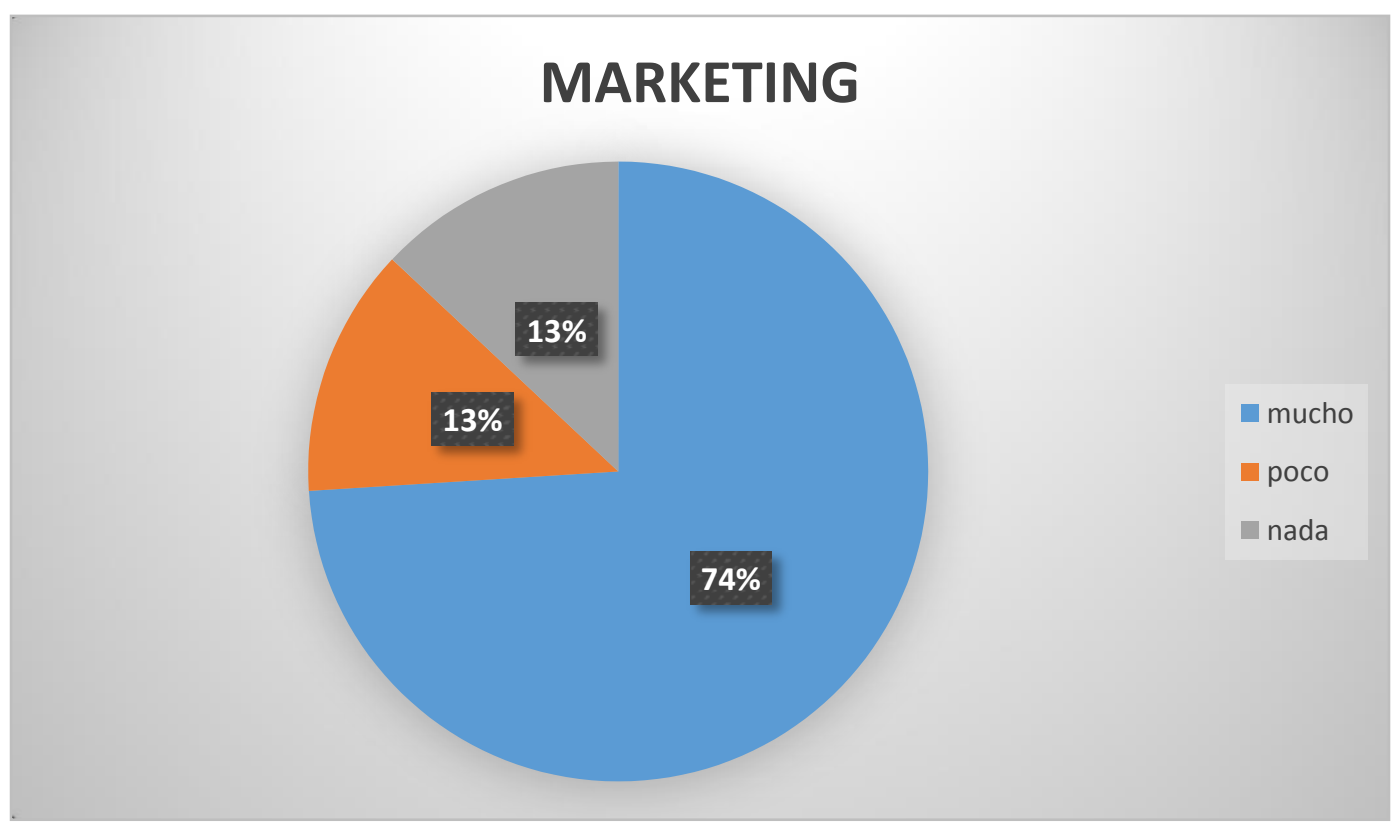

\section{Gráfico 7 Marketing}

Fuente: Parroquia Camilo Ponce Enríquez

Análisis de datos En la encuesta aplicada a los directivos y empleados de la coordinación zona cinco del Mies, relacionada con nueva gestión de marketing, los resultados obtenidos demuestran que un 74\% de los encuestados manifestaron que es necesario una nueva gestión 
marketing, por lo que se puede interpretar que es necesario actualizar o direccionar una nueva gestión de marketing.

8.- ¿Cree Usted estar dispuesto a capacitarse en las buenas prácticas de marketing?

\begin{tabular}{|l|l|l|}
\hline \multicolumn{1}{|c|}{ alternativas } & \multicolumn{1}{c|}{$\mathbf{N}^{\circ}$ encuestados } & \multicolumn{1}{c|}{ porcentaje } \\
\hline Mucho & 22 & 74 \\
\hline Poco & 4 & 13 \\
\hline Nada & 1 & 13 \\
\hline TOTAL & 27 & 100 \\
\hline
\end{tabular}

Cuadro 8

Fuente: Parroquia Camilo Ponce Enríquez

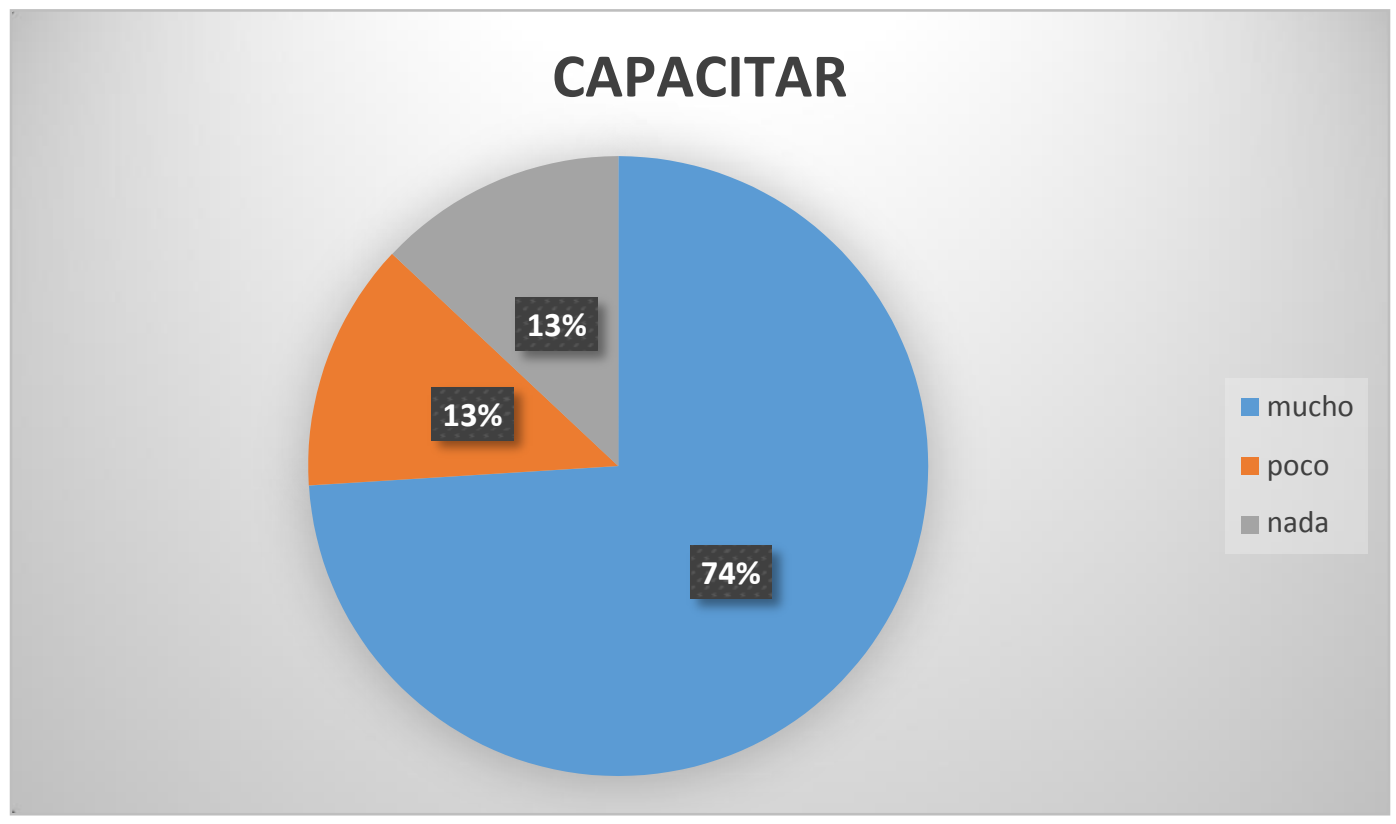

\section{Gráfico 8 Capacitor}

Fuente: Parroquia Camilo Ponce Enríquez

Análisis de datos En la encuesta aplicada a los directivos y empleados de la coordinación zonal cinco del Mies, relacionada con la capacitación de las buenas prácticas de marketing, los resultados obtenidos demuestran que un $74 \%$ de los encuestados consideran estar dispuestos hacer 
capacitados en las buenas prácticas de marketing, por lo que se puede interpretar que la actitud y predisposición en los directivos y empleados es positiva hacia un mejoramiento.

\section{9.- ¿Conoce usted lo que es políticas de marketing públicas?}

\begin{tabular}{|l|l|l|}
\hline \multicolumn{1}{|c|}{ alternativas } & \multicolumn{1}{c|}{$\mathbf{N}^{\circ}$ encuestados } & \multicolumn{1}{c|}{ porcentaje } \\
\hline Mucho & 3 & 74 \\
\hline Poco & 3 & 13 \\
\hline Nada & 21 & 13 \\
\hline TOTAL & 27 & 100 \\
\hline
\end{tabular}

Cuadro 9

Fuente: Parroquia Camilo Ponce Enríquez

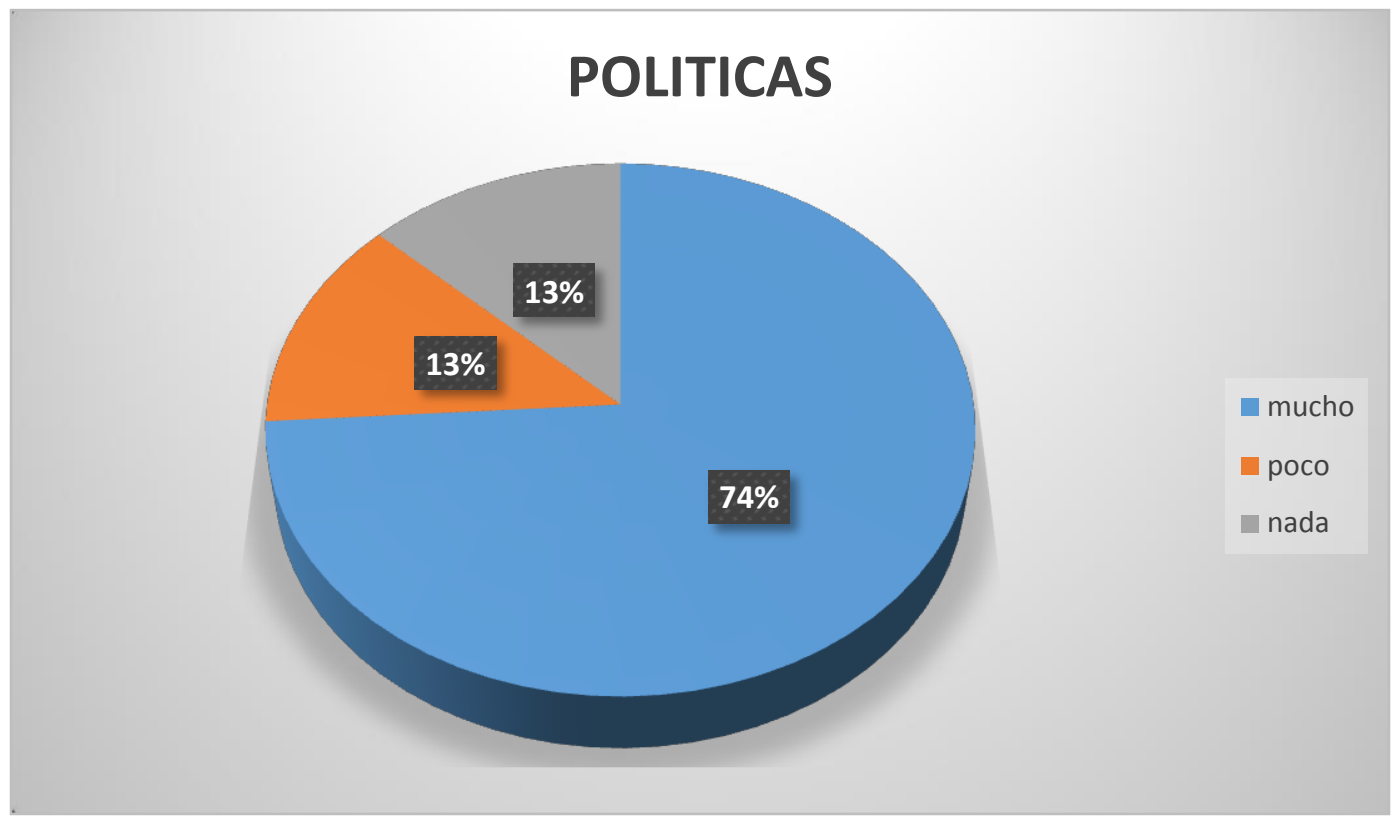

\section{Gráfico 9 Políticas}

Fuente: Parroquia Camilo Ponce Enríquez

Análisis de datos En la encuesta aplicada a los directivos y empleados de la coordinación zonal cinco del Mies, relacionada con políticas de marketing públicas, los resultados obtenidos demuestran que un 74\% de los encuestados desconocen todo lo relacionado con políticas públicas de 
marketing, por lo que se puede interpretar que es necesario involucrar a los empleados en el conocimiento de las políticas públicas de marketing.

10.- ¿Participaría Usted activamente en la aplicación de políticas de marketing públicas en la Coordinación Zonal cinco del Mies?

\begin{tabular}{|l|l|l|}
\hline \multicolumn{1}{|c|}{ alternativas } & \multicolumn{1}{c|}{$\mathbf{N}^{\circ}$ encuestados } & \multicolumn{1}{c|}{ porcentaje } \\
\hline Mucho & 15 & 74 \\
\hline Poco & 5 & 13 \\
\hline Nada & 7 & 13 \\
\hline TOTAL & 27 & 100 \\
\hline
\end{tabular}

Cuadro 10

Fuente: Parroquia Camilo Ponce Enríquez

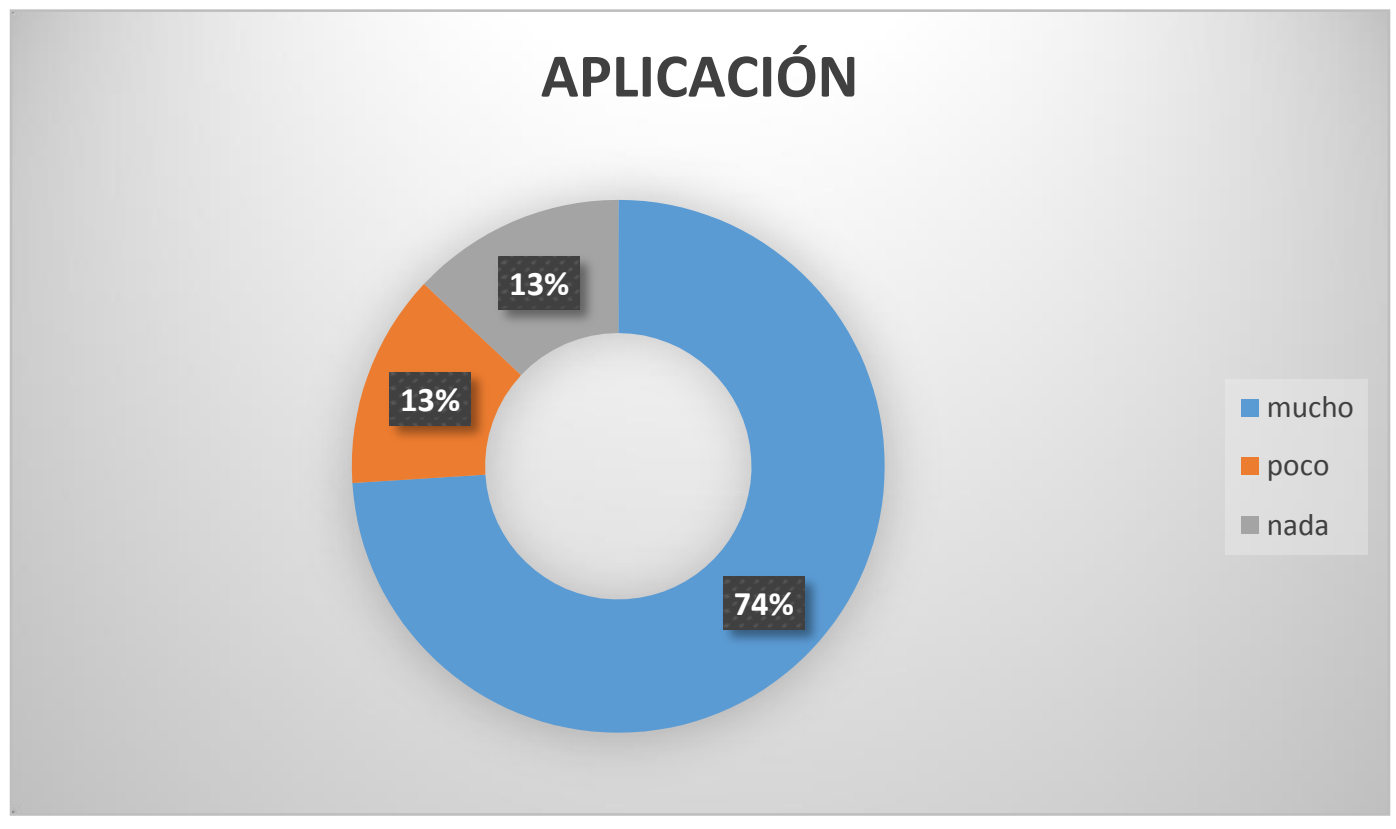

Gráfico 10 Aplicación

Fuente: Parroquia Camilo Ponce Enríquez

Análisis de datos En la encuesta aplicada a los directivos y empleados de la coordinación zonal cinco del Mies, relacionada con la participación en la aplicación de políticas de marketing, los 
resultados obtenidos demuestran que un $74 \%$ de los encuestados se encuentran predispuesto en aplicar las políticas de marketing, por lo que se puede interpretar que es necesario involucrar a los empleados en la participación de políticas de marketing.

11.- ¿Cree Usted que el área en la que labora se beneficiaría de las políticas de marketing públicas?

\begin{tabular}{|l|l|l|}
\hline alternativas & $\mathbf{N}^{\circ}$ encuestados & porcentaje \\
\hline Mucho & 16 & 74 \\
\hline Poco & 5 & 13 \\
\hline Nada & 6 & 13 \\
\hline TOTAL & 27 & 100 \\
\hline
\end{tabular}

Cuadro 11

Fuente: Parroquia Camilo Ponce Enríquez

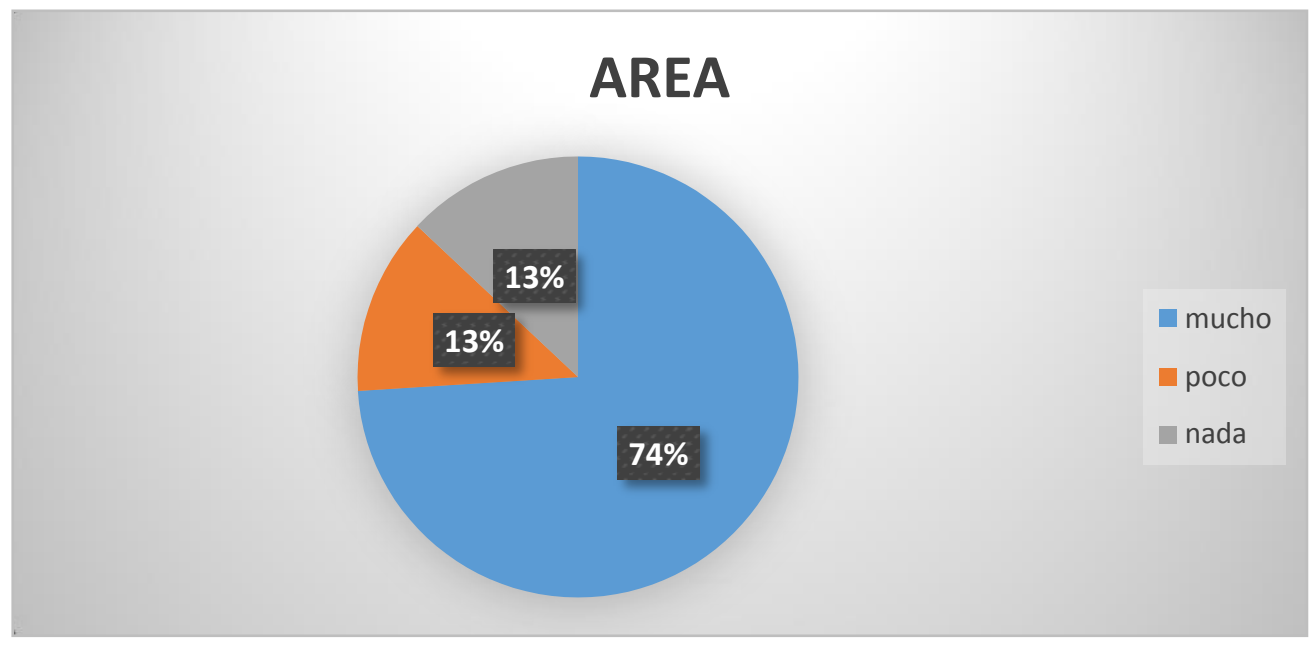

Gráfico 11 Área

Fuente: Parroquia Camilo Ponce Enríquez 
Análisis de datos En la encuesta aplicada a los directivos y empleados de la coordinación zonal cinco del Mies, relacionada con el beneficio del área laboral si se aplicaría las políticas de marketing, los resultados obtenidos demuestran que un $74 \%$ de los encuestados consideran que sus áreas de trabajo se beneficiarían con las nuevas políticas de marketing, por lo que se puede interpretar que existe una buena predisposición de mejorar los procesos comunicacionales basado en las políticas de marketing.

\section{2.- ¿Creé usted que la Coordinación Zonal cinco del Mies mejoraría su nivel de imagen con la} aplicación de las políticas de marketing públicas?

\begin{tabular}{|l|l|l|}
\hline Alternativas & $\mathbf{N}^{\circ}$ encuestados & porcentaje \\
\hline Mucho & 21 & 74 \\
\hline Poco & 5 & 13 \\
\hline Nada & 1 & 13 \\
\hline TOTAL & 27 & 100 \\
\hline
\end{tabular}

Cuadro 12

Fuente: Parroquia Camilo Ponce Enríquez

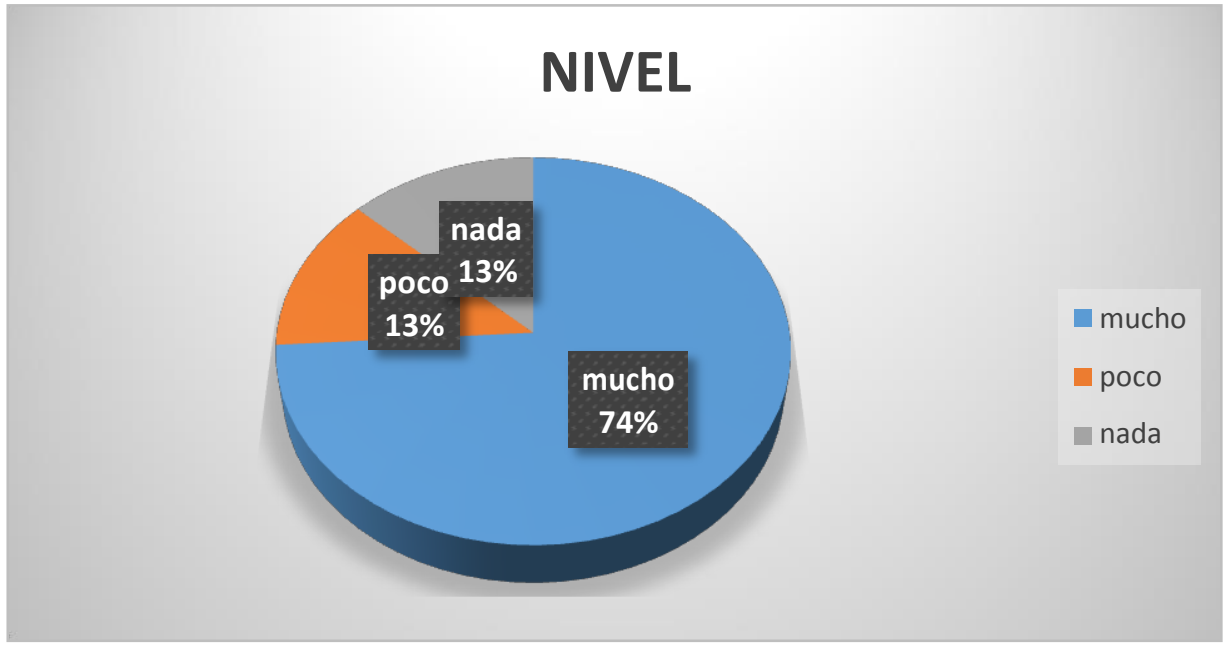

Gráfico 12 Nivel

Fuente: Parroquia Camilo Ponce Enríquez 
Análisis de datos En la encuesta aplicada a los directivos y empleados de la coordinación zonal cinco del Mies, relacionada si mejoraría su nivel de imagen con las políticas públicas de marketing, los resultados obtenidos demuestran que un $74 \%$ de los encuestados consideran que la imagen institucional se mejoraría, por lo que se puede interpretar que la implementación de dichas políticas de marketing públicos tendría éxitos.

Encuesta a los usuarios de la Coordinación Zonal cinco del Ministerio de Inclusión Económica y Social.

\section{1.- ¿Qué tipo de información de las entidades públicas le interesa más?}

\begin{tabular}{|l|l|l|}
\hline \multicolumn{1}{|c|}{ alternativas } & $\mathbf{N}^{\circ}$ encuestados & porcentaje \\
\hline Trámites y requisitos & $\mathbf{3 5}$ & $\mathbf{6 4}$ \\
\hline actividades & $\mathbf{2}$ & $\mathbf{4}$ \\
\hline presupuesto & $\mathbf{0}$ & $\mathbf{0}$ \\
\hline Compras y Licitaciones & $\mathbf{2}$ & $\mathbf{4}$ \\
\hline Otros & $\mathbf{1 0}$ & $\mathbf{1 9}$ \\
\hline No sabe/no contesta & $\mathbf{5}$ & $\mathbf{9}$ \\
\hline TOTAL & $\mathbf{5 4}$ & $\mathbf{1 0 0}$ \\
\hline
\end{tabular}

\section{Cuadro 13}

Fuente: Parroquia Camilo Ponce Enríquez 


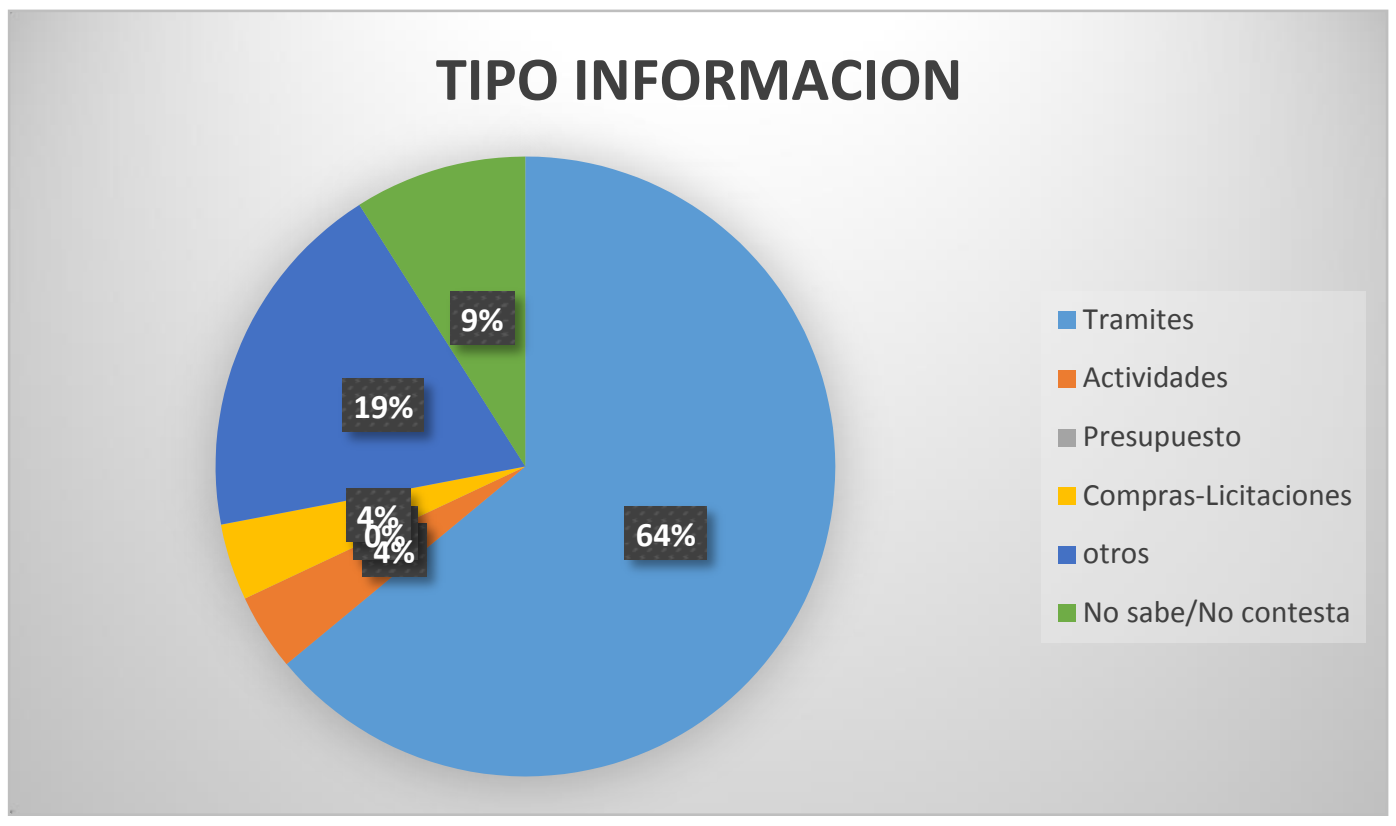

\section{Gráfico 13 Tipo de información}

Fuente: Parroquia Camilo Ponce Enríquez

Análisis de datos En la encuesta aplicada a los usuarios de la coordinación zonal cinco del Mies, relacionada con el tipo de información que le interesa realizar en el Mies, los resultados obtenidos demuestran que un $64 \%$ de los encuestados realizan trámites y requisitos, por lo que se puede interpretar que los usuarios hacen uso de los productos/servicios por lo que es necesario la atención bien del usuario.

2.- ¿En los últimos doce meses, ¿ha realizado algún trámite en entidades públicas?

\begin{tabular}{|l|l|l|}
\hline \multicolumn{1}{|c|}{ Alternativas } & $\mathbf{N}^{\circ}$ encuestados & porcentaje \\
\hline $\mathrm{Si}$ & $\mathbf{5 0}$ & $\mathbf{9 2}$ \\
\hline No & $\mathbf{2}$ & $\mathbf{4}$ \\
\hline No sabe/no contesta & $\mathbf{2}$ & $\mathbf{4}$ \\
\hline TOTAL & $\mathbf{5 4}$ & $\mathbf{1 0 0}$ \\
\hline
\end{tabular}

\section{Cuadro 14}

Fuente: Parroquia Camilo Ponce Enríquez 


\section{TRAMITE}

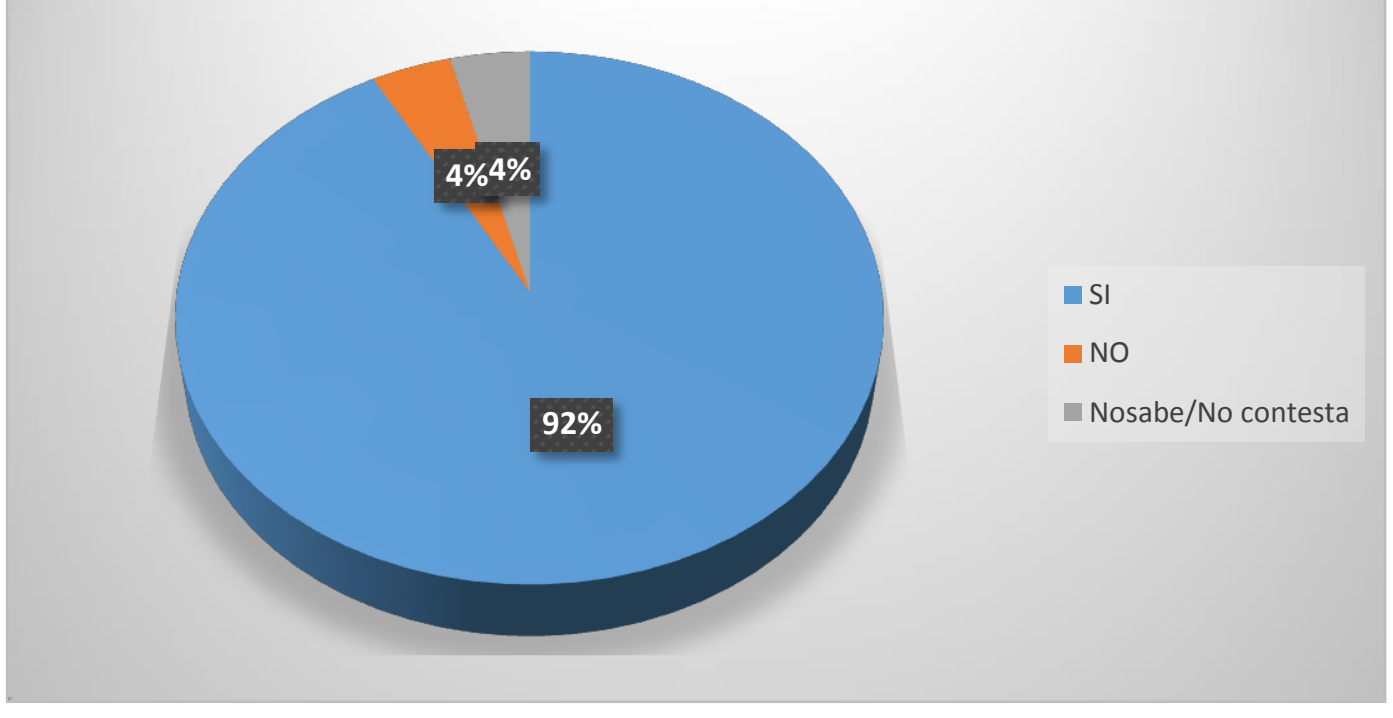

\section{Gráfico 14 Trámite}

Fuente: Parroquia Camilo Ponce Enríquez

Análisis de datos En la encuesta aplicada a los usuarios de la coordinación zonal cinco del Mies, relacionada si ha realizado tramites en los últimos doce meses, los resultados obtenidos demuestran que un $92 \%$ de los encuestados han realizado tramites los últimos doce meses, por lo que se puede interpretar que la implementación de los procesos de comunicación es fundamental en la institución para conocer las necesidades de los usuarios. 
3.- ¿Cuál es el trámite más complicado y burocrático que ha realizado durante los últimos $\mathbf{1 2}$ meses en alguna entidad pública? (sólo para los que contestaron "sí" en la pregunta 2)

\begin{tabular}{|l|l|l|}
\hline Alternativas & $\mathbf{N}^{\circ}$ encuestados & porcentaje \\
\hline Municipal & $\mathbf{1 0}$ & $\mathbf{2 0}$ \\
\hline Judicial & $\mathbf{5}$ & $\mathbf{1 0}$ \\
\hline De salud & $\mathbf{1}$ & $\mathbf{2}$ \\
\hline Policial & $\mathbf{3}$ & $\mathbf{6}$ \\
\hline Banco Fomento & $\mathbf{1 2}$ & $\mathbf{2 4}$ \\
\hline Mies & $\mathbf{1 9}$ & $\mathbf{3 8}$ \\
\hline TOTAL & $\mathbf{5 0}$ & $\mathbf{1 0 0}$ \\
\hline
\end{tabular}

Cuadro 15

Fuente: Parroquia Camilo Ponce Enríquez

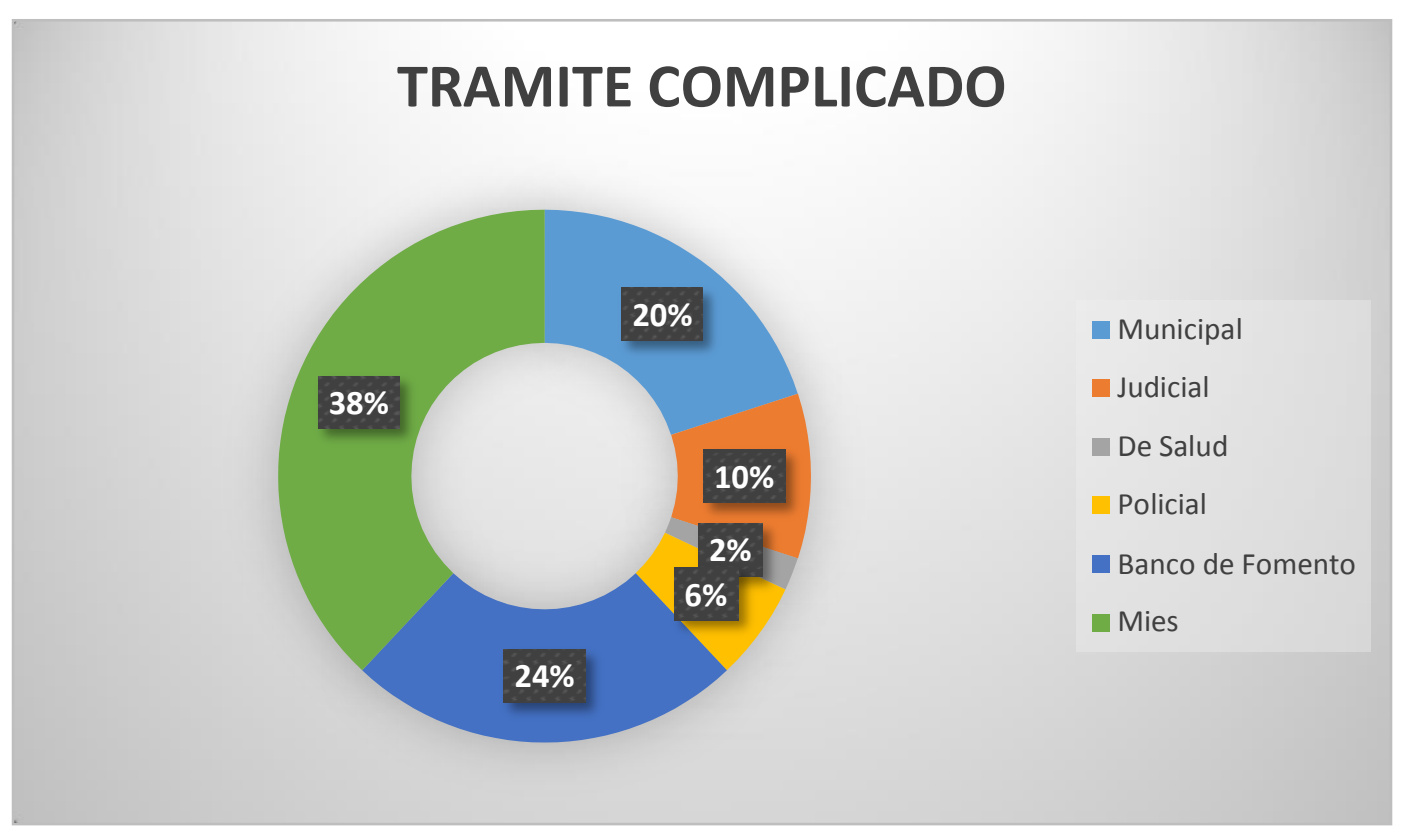

\section{Gráfico 15 Trámite complicado}

Fuente: Parroquia Camilo Ponce Enríquez 
Análisis de datos En la encuesta aplicada a los usuarios de la coordinación zonal cinco del Mies, relacionada con el trámite más complicado y burocrático en las instituciones públicas, los resultados obtenidos demuestran que un $38 \%$ de los encuestados consideran que el Mies y $20 \%$ el Municipio es más complicado y burocrático, por lo que se puede interpretar que es necesario aplicar buenas prácticas de marketing para comunicar los procesos.

4.- En los últimos doce meses, ¿ha hecho excesiva, bastante, poca o nada de "cola" para hacer gestiones en entidades públicas? (sólo para los que contestaron "sí" en la pregunta 2)

\begin{tabular}{|l|l|l|}
\hline \multicolumn{1}{|c|}{ Alternativas } & $\mathbf{N}^{\circ}$ encuestados & porcentaje \\
\hline Excesiva & $\mathbf{2 0}$ & $\mathbf{4 0}$ \\
\hline Bastante & $\mathbf{1 5}$ & $\mathbf{3 0}$ \\
\hline Poca & $\mathbf{8}$ & $\mathbf{1 6}$ \\
\hline No sabe/No contesta & $\mathbf{7}$ & $\mathbf{1 4}$ \\
\hline TOTAL & $\mathbf{5 0}$ & $\mathbf{1 0 0}$ \\
\hline
\end{tabular}

Cuadro 16

Fuente: Parroquia Camilo Ponce Enríquez 


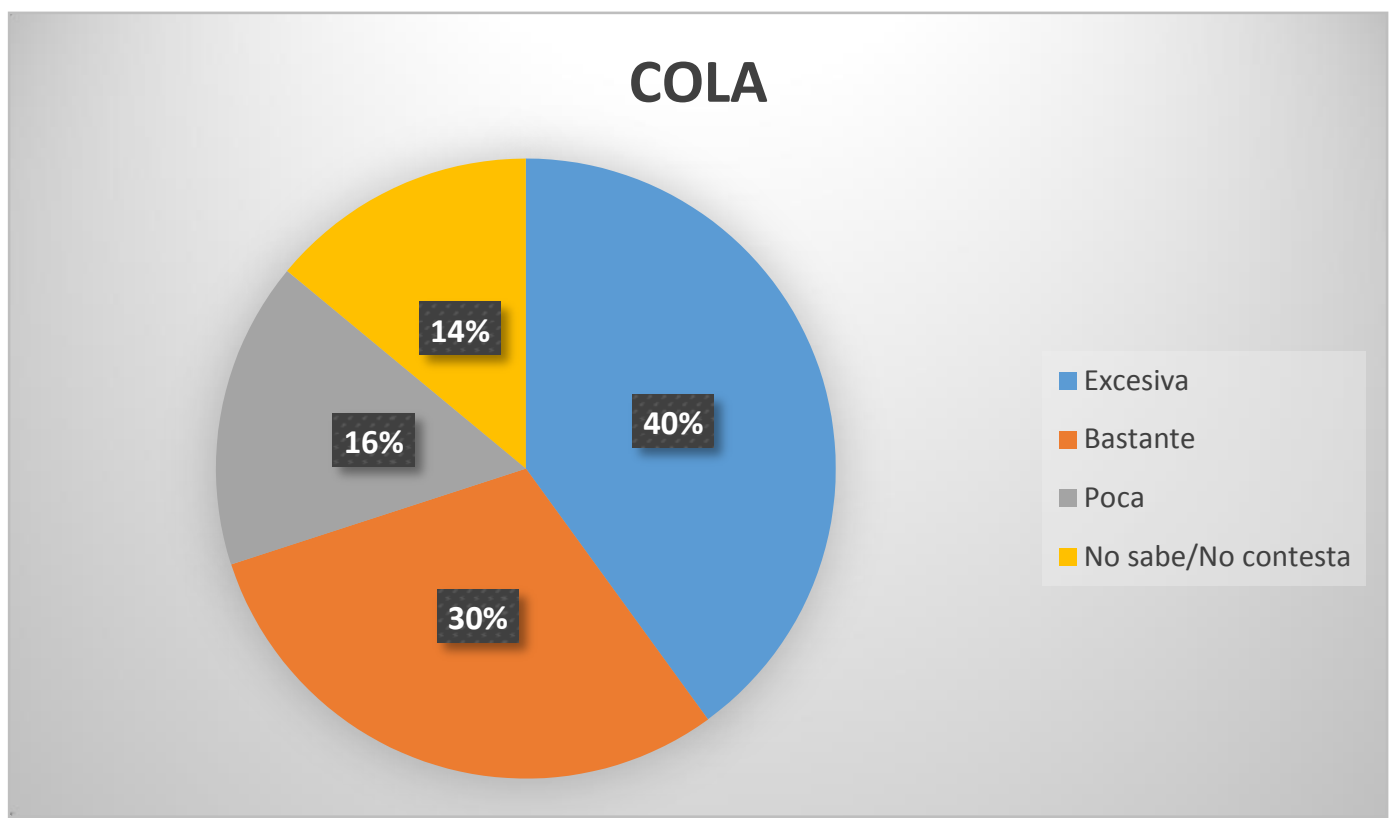

\section{Gráfico 16 Cola}

Fuente: Parroquia Camilo Ponce Enríquez

Análisis de datos En la encuesta aplicada a los usuarios de la coordinación zonal cinco del Mies, relacionada con el tiempo de espera en la cola, los resultados obtenidos demuestran que un $40 \%$ de los encuestados consideran que el tiempo en cola es excesiva y un $30 \%$ es bastante, por lo que se puede interpretar que es necesario disminuir tiempo en cola.

\section{5.- ¿Qué es lo que más le incomoda en una gestión ante una entidad pública? (respuesta} asistida)

\begin{tabular}{|l|l|l|}
\hline \multicolumn{1}{|c|}{ alternativas } & $\mathbf{N}^{\circ}$ encuestados & porcentaje \\
\hline Tiempo de espera & $\mathbf{1 2}$ & $\mathbf{2 2}$ \\
\hline Trato del personal & $\mathbf{1 1}$ & $\mathbf{2 0}$ \\
\hline Falta de información & $\mathbf{1 3}$ & $\mathbf{2 4}$ \\
\hline Exceso de requisito & $\mathbf{1 2}$ & $\mathbf{2 2}$ \\
\hline
\end{tabular}




\begin{tabular}{|l|l|l|}
\hline Local inapropiado & $\mathbf{2}$ & $\mathbf{4}$ \\
\hline No sabe/ No contesta & $\mathbf{4}$ & $\mathbf{8}$ \\
\hline TOTAL & $\mathbf{5 4}$ & $\mathbf{1 0 0}$ \\
\hline
\end{tabular}

\section{Cuadro 17}

Fuente: Parroquia Camilo Ponce Enríquez

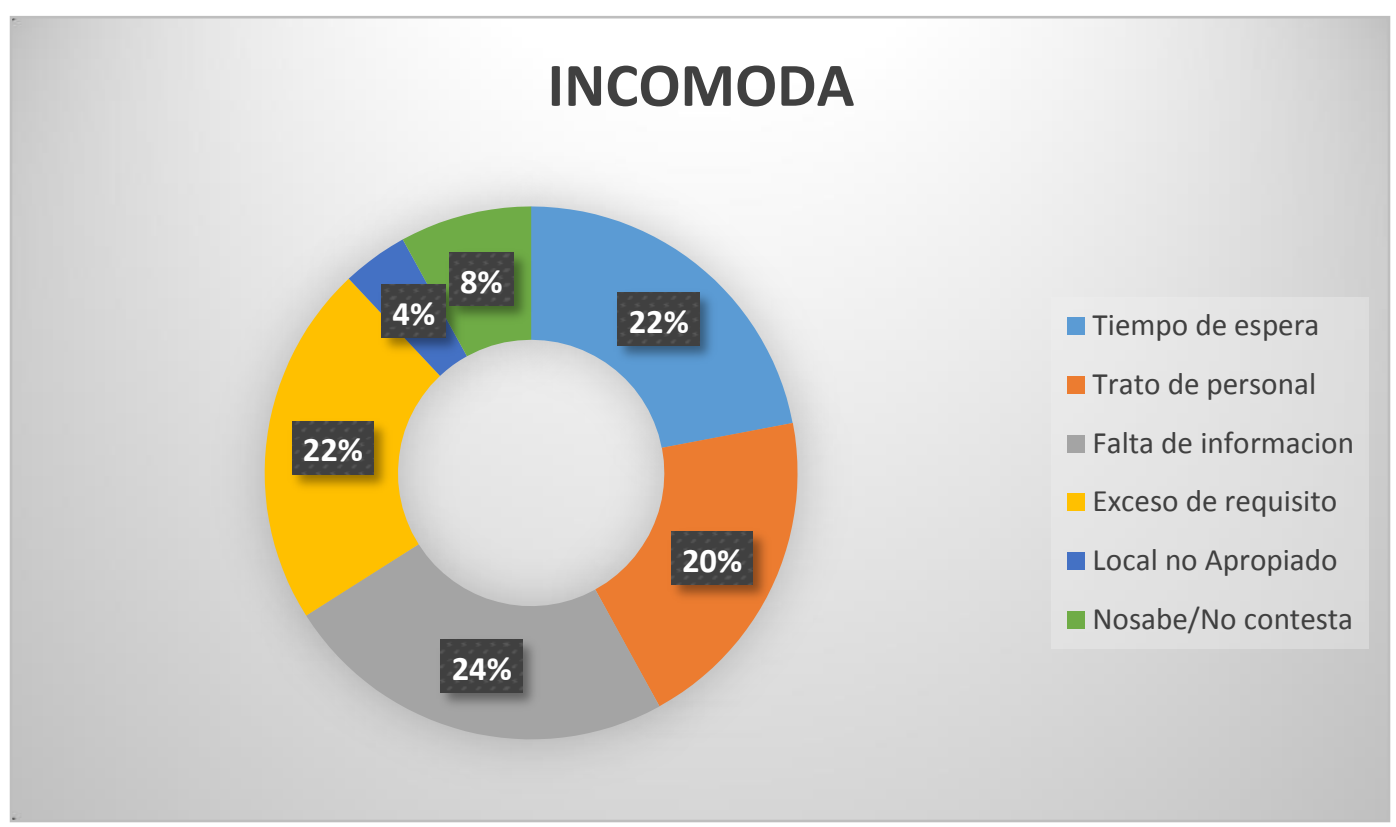

\section{Gráfico 17 Incomoda}

Fuente: Parroquia Camilo Ponce Enríquez

Análisis de datos En la encuesta aplicada a los usuarios de la coordinación zonal cinco del Mies, relacionada con lo que más le incomoda en una gestión, los resultados obtenidos demuestran que un $24 \%$ de los encuestados consideran que la falta de información y un $22 \%$ en tiempo de espera y requisitos respectivamente, por lo que se puede interpretar que la información debe ser maneja de mejor manera e informar a tiempo. 
6.- ¿Sabe Usted que los formularios para los trámites en las entidades públicas son gratuitos?

\begin{tabular}{|l|l|l|}
\hline \multicolumn{1}{|c|}{ alternativas } & $\mathbf{N}^{\circ}$ encuestados & porcentaje \\
\hline $\mathrm{Si}$ & $\mathbf{2 7}$ & $\mathbf{5 0}$ \\
\hline No & $\mathbf{2 2}$ & $\mathbf{4 1}$ \\
\hline No sabe/No contesta & $\mathbf{5}$ & $\mathbf{9}$ \\
\hline TOTAL & $\mathbf{5 4}$ & $\mathbf{1 0 0}$ \\
\hline
\end{tabular}

Cuadro 18

Fuente: Parroquia Camilo Ponce Enríquez

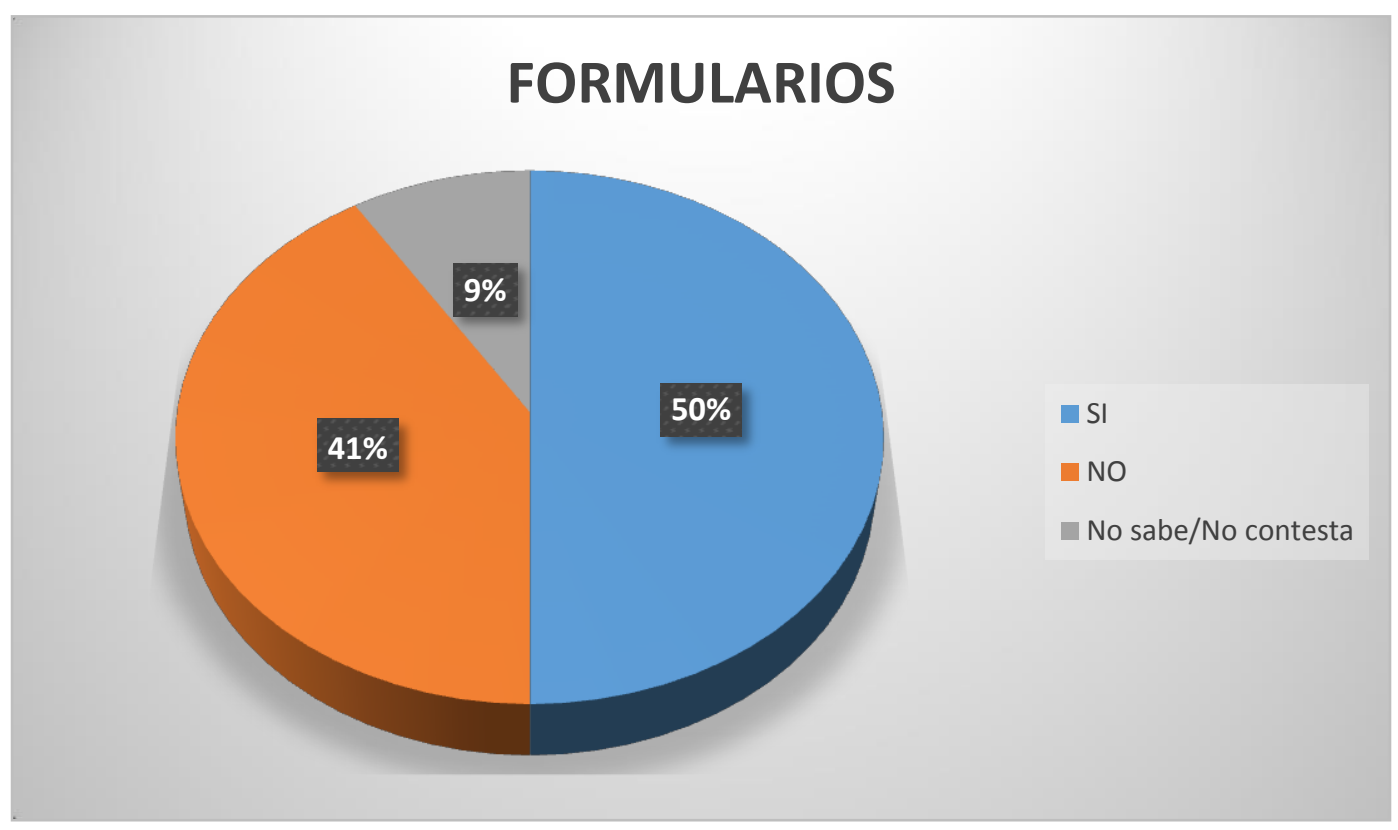

Gráfico 18 Formularios

Fuente: Parroquia Camilo Ponce Enríquez

Análisis de datos En la encuesta aplicada a los usuarios de la coordinación zonal cinco del Mies, relacionada con la gratuidad de los formularios en los trámites, los resultados obtenidos demuestran que un $50 \%$ de los encuestados conocen de la gratuidad y un $41 \%$ desconocen la 
gratuidad, por lo que se puede interpretar que es necesario hacer conocer la existencia de que los tramites son gratuitos en las instituciones públicas.

7.- En general, ¿considera que en los últimos años la atención a los ciudadanos en las instituciones públicas, se mantiene igual o ha empeorado?

\begin{tabular}{|l|l|l|}
\hline \multicolumn{1}{|c|}{ alternativas } & $\mathbf{N}^{\circ}$ encuestados & porcentaje \\
\hline Ha mejorado & $\mathbf{1 8}$ & $\mathbf{3 3}$ \\
\hline Se mantiene igual & $\mathbf{2 0}$ & $\mathbf{3 7}$ \\
\hline Ha empeorado & $\mathbf{1 2}$ & $\mathbf{2 2}$ \\
\hline No sabe/ No contesta & $\mathbf{4}$ & $\mathbf{8}$ \\
\hline TOTAL & $\mathbf{5 4}$ & $\mathbf{1 0 0}$ \\
\hline
\end{tabular}

\section{Cuadro 19}

Fuente: Parroquia Camilo Ponce Enríquez

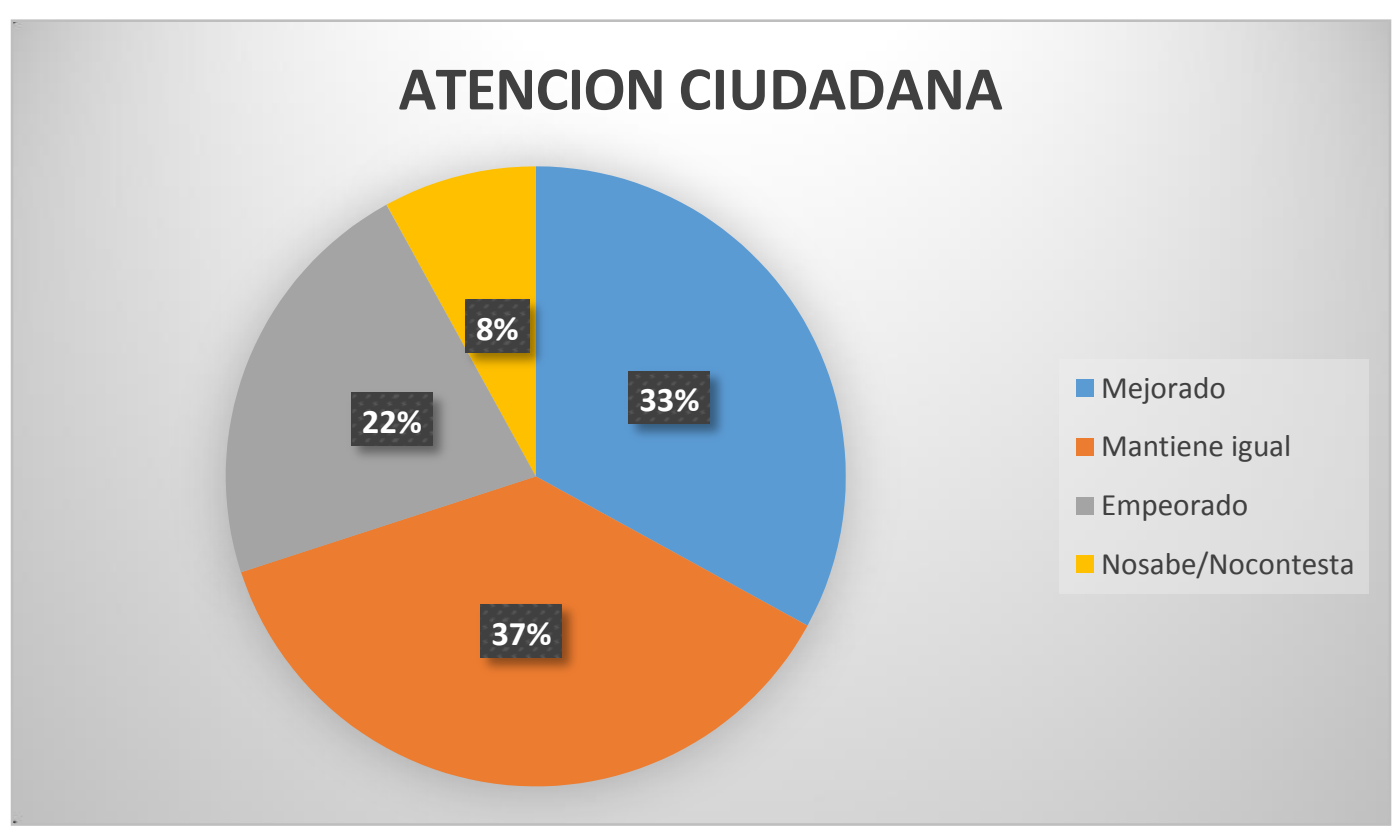

Gráfico 19 Atención ciudadana

Fuente: Parroquia Camilo Ponce Enríquez 
Análisis de datos En la encuesta aplicada a los usuarios de la coordinación zonal cinco del Mies, relacionada con la atención a los ciudadanos en las instituciones públicas, los resultados obtenidos demuestran que un $37 \%$ de los encuestados consideran que la atención al usuario se mantiene igual y un $22 \%$ ha empeorado, por lo que se puede interpretar que es necesario mejorar las buenas prácticas de marketing en la atención del usuario.

\section{8.- En general, ¿cuál es la principal virtud que tienen los trabajadores de las entidades} públicas? (respuesta asistida)

\begin{tabular}{|l|l|l|}
\hline \multicolumn{1}{|c|}{ Alternativas } & $\mathbf{N}^{\circ}$ encuestados & porcentaje \\
\hline Buen trato & 6 & 11 \\
\hline Responsabilidad & 5 & 9 \\
\hline Dedicación al trabajo & 3 & 6 \\
\hline Honestidad & 5 & 9 \\
\hline Ninguna & 35 & 65 \\
\hline TOTAL & 54 & 100 \\
\hline
\end{tabular}

Cuadro 20

Fuente: Parroquia Camilo Ponce Enríquez 


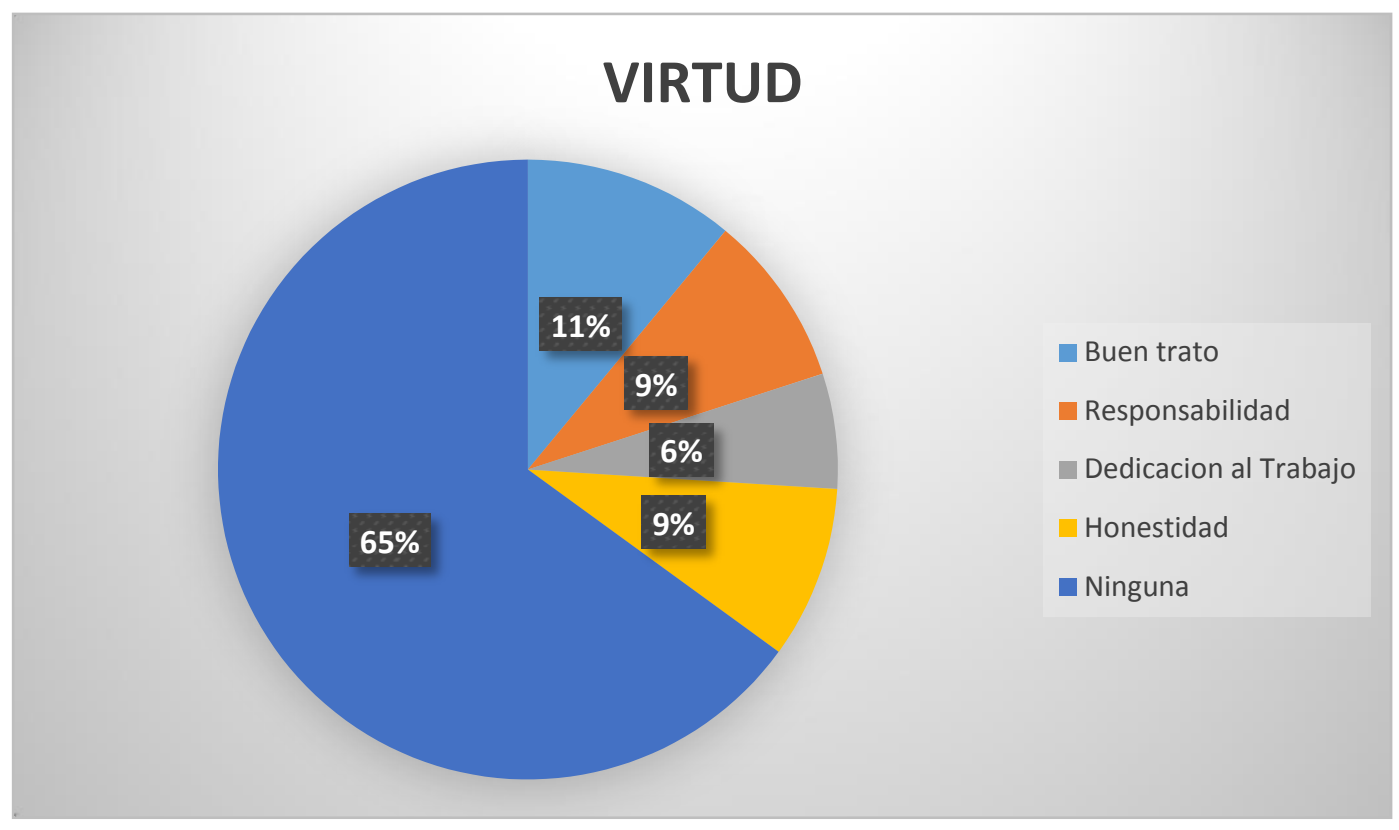

\section{Gráfico 20}

Fuente: Parroquia Camilo Ponce Enríquez

Análisis de datos En la encuesta aplicada a los usuarios de la coordinación zonal cinco del Mies, relacionada con la virtud principal de los empleados de las instituciones públicas, los resultados obtenidos demuestran que un $65 \%$ de los encuestados consideran que los empleados no tienen ninguna virtud con los ciudadanos que asisten a las instituciones públicas por un servicio, por lo que se puede interpretar que es fundamental diseñar políticas de buenas prácticas de Marketing basadas en las virtudes principales de los empleados.

\section{9.- ¿y cuál es el principal defecto que tienen los trabajadores de las entidades públicas?}

\begin{tabular}{|l|l|l|}
\hline \multicolumn{1}{|c|}{ alternativas } & $\mathbf{N}^{\circ}$ encuestados & porcentaje \\
\hline Mal trato & $\mathbf{2 1}$ & $\mathbf{3 9}$ \\
\hline Irresponsabilidad & $\mathbf{3}$ & $\mathbf{6}$ \\
\hline Impuntualidad & $\mathbf{5}$ & $\mathbf{9}$ \\
\hline Falta de dedicación al trabajo & $\mathbf{2 2}$ & $\mathbf{4 0}$ \\
\hline Ninguna & $\mathbf{3}$ & $\mathbf{6}$ \\
\hline
\end{tabular}


Buenas prácticas de marketing del sector privado y su incidencia en la gestión pública MIES

\begin{tabular}{|l|l|l|}
\hline TOTAL & 54 & 100 \\
\hline
\end{tabular}

Cuadro 21

Fuente: Parroquia Camilo Ponce Enríquez

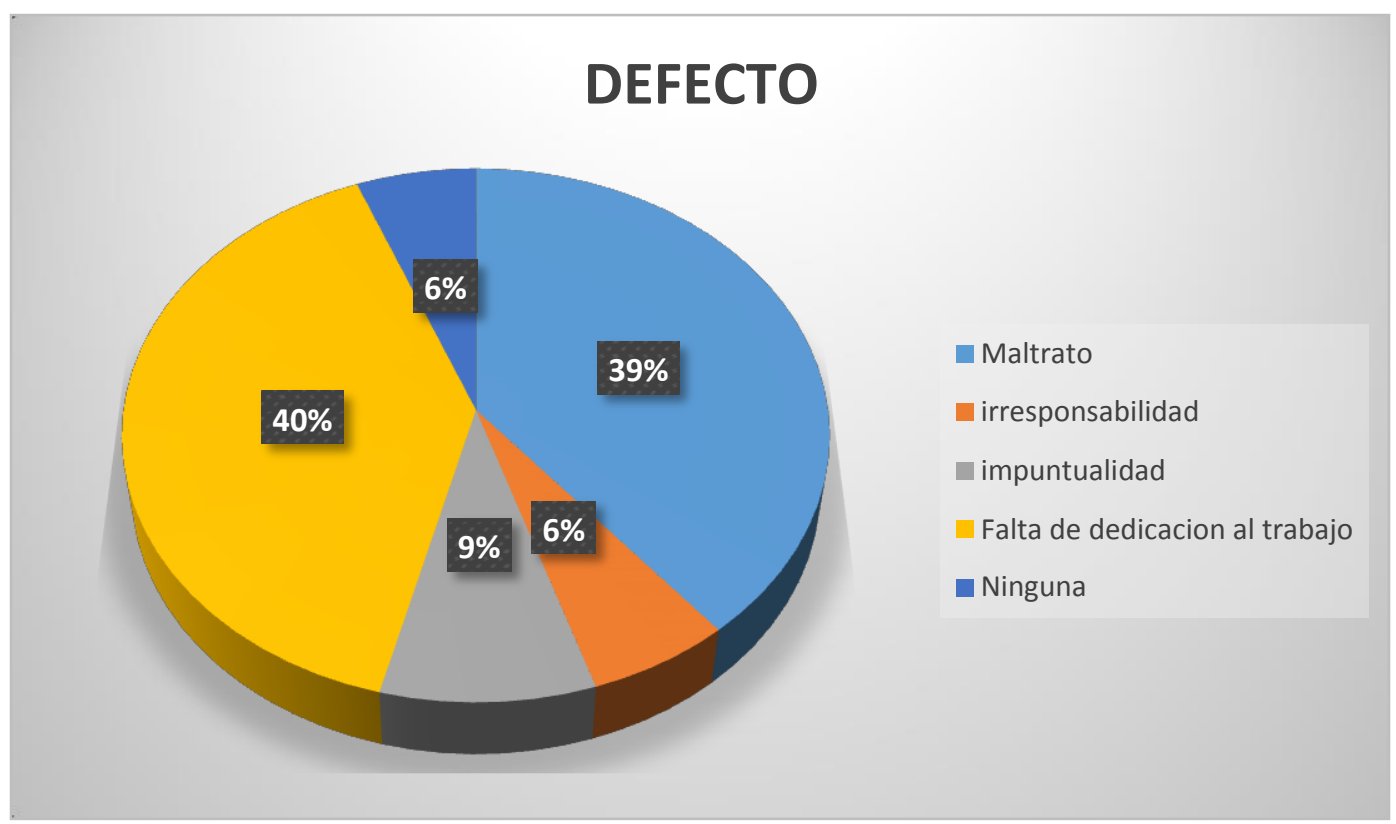

\section{Gráfico 21 Defecto}

Fuente: Parroquia Camilo Ponce Enríquez

Análisis de datos En la encuesta aplicada a los usuarios de la coordinación zonal cinco del Mies, relacionada con los defectos que tienen los empleados de las instituciones públicas, los resultados obtenidos demuestran que un $40 \%$ de los encuestados consideran que la falta de dedicación al trabajo y 39\% al maltrato, por lo que se puede interpretar que es necesario fortalecer políticas de buenas prácticas de marketing enfocando la conducta. 


\section{0.- ¿Cree Usted que la atención a los ciudadanos en el MIES es?}

\begin{tabular}{|l|l|l|}
\hline alternativas & $\mathbf{N}^{\circ}$ encuestados & Porcentaje \\
\hline Muy buena & 4 & 8 \\
\hline Buena & 9 & 17 \\
\hline Regular & 10 & 19 \\
\hline Mala & 24 & 44 \\
\hline Muy mala & 7 & 12 \\
\hline TOTAL & 54 & 100 \\
\hline
\end{tabular}

Cuadro 22

Fuente: Parroquia Camilo Ponce Enríquez

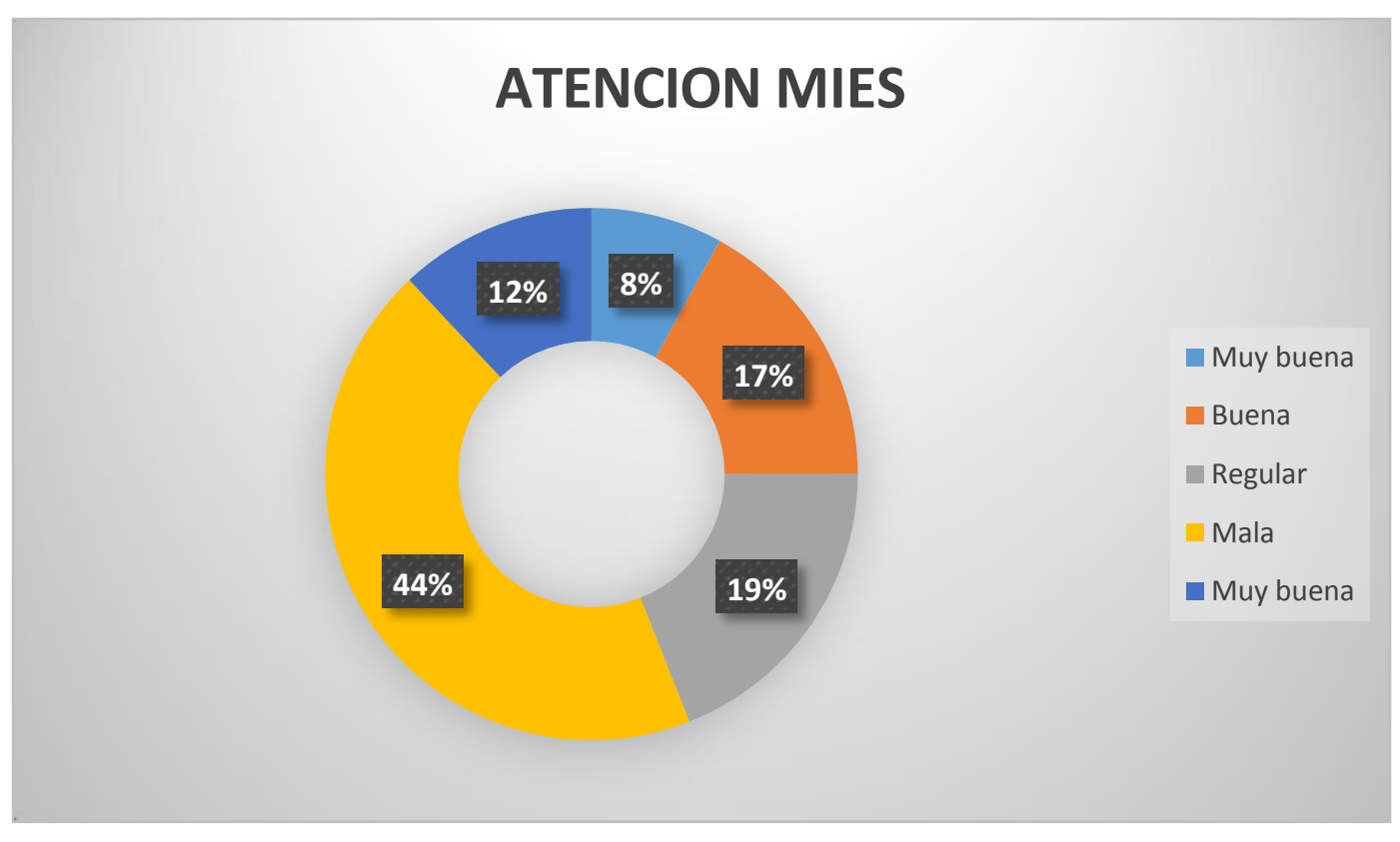

Gráfico 22 Atención MIES

Fuente: Parroquia Camilo Ponce Enríquez

Análisis de datos En la encuesta aplicada a los usuarios de la coordinación zonal cinco del Mies, relacionada con la atención de los ciudadanos en el Mies, los resultados obtenidos demuestran 
Buenas prácticas de marketing del sector privado y su incidencia en la gestión pública MIES

que un $44 \%$ de los encuestados consideran que es mala la atención en el Mies y un 19\% es regular, por lo que se puede interpretar que no se aplica las políticas de buenas prácticas de Marketing.

\section{Conclusiones}

El nivel de conocimiento sobre la gestión de marketing basado en las Buenas Prácticas de Marketing del sector empresarial privado, su aplicación en la coordinación zonal cinco del Ministerio de Inclusión económica y social de parte de los directivos, empleados y trabajadores es prácticamente nula.

Los empleados y directivos de la coordinación zonal cinco del Ministerio de Inclusión Económica y Social han entendido que la gestión de Marketing ayuda al proceso de vinculación con los usuarios, por lo tanto, es conveniente diseñar políticas de marketing.

En cuanto a los procesos comunicacionales interno, en la coordinación zonal cinco del Ministerio no existe interrelación, por lo que es necesario implementar políticas de buenas prácticas de marketing para ser vinculadas en las diferentes áreas de la coordinación.

Los usuarios tienen una percepción negativa sobre la falta de información y requisitos para los servicios que se dispensa en las instituciones públicas.

El modelo intervenido del marketing social es el que nosotros hemos elegido para el estudio del servicio público deportivo fundamentado en su consolidada base científica y en su capacidad técnica y de procedimiento para incidir -desde las técnicas del intercambio social- en el comportamiento social manifestado a través del deporte. 
No obstante, nos gustaría que se considerara la diferencia entre la existencia de un marketing para el servicio público diferenciado de otros servicios dado que su producción y comercialización con lleva una presentación del servicio de forma diferente.

\section{Bibliografía.}

Arellano, R. (2007). Marketing: Enfoque Americalatina. México: McGraw Hill.

Bagozzi, R. (2006). Teoría General del Marking. México: Prentice Hall.

Birgitta, L. (2008). Gestión del conocimiento. México: Prentice Hall.

Esparcia, J. (2009). Planeamiento Estratégica. Bogotá: Legis.

GARCIA, M. (2009). Proceso Administrativo. México: Trillas.

Heinrich, B. (2005). Capital Humano, Capital Social y Conocimiento. México: Prentice Hall.

Hernández, A. (2001). el espacio de la política en la gestión pública. Ambato: Uniandes.

Klingner, D. (2002). La administración del personal en el Sector Publico. México: McGraw Hill.

Kotler, P. (2000). Fundamentos de Marketing. México: Prentice Hall.

Kotler, P. (2004). Marketing de servicio. México: McGraw Hill.

Kotler, P. (2005). Visión del Marketing. México: McGraw Hill.

Ochoa, H. (2007). Gestión Administrativa. Bogotá: Legis.

Parasuman, A. (2006). Modelo conceptual calidad de servicio. México: McGraw Hill.

Quintana, F. (2002). Marketing Público. Madrid: Síntesis.

Universidad los andes. (2001). introducción a la teoría de sistemas. Bogotá: Universitaria.

Wayne, M. (2006). Administración de Recursos Humanos. Madrid: prentice Hall. 